NASA CONTRACTOR REPORT

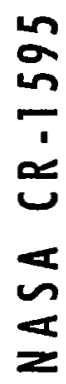

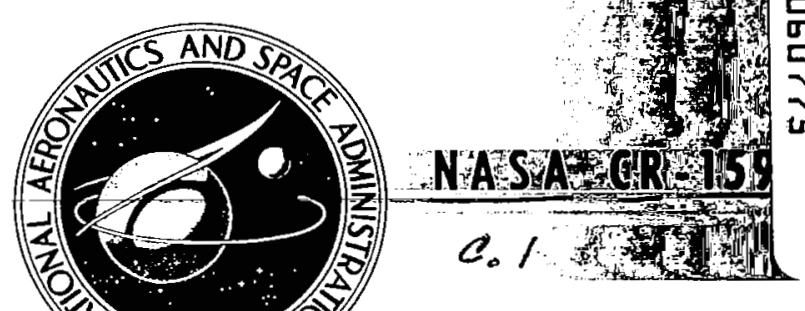

INOAN COPY: RETURN TO AFWL (WLOL)

RIRTIAND ATrB, N MEKK

\title{
RECIRCULATION PAT'TERNS \\ IN THE INITIAL REGION OF \\ COAXIAL JETS
}

by Tzvi Rozenman and Herbert Weinstein

Prepared by

ILLINOIS INSTITUTE OF TECHNOLOGY

Chicago, III.

for Lewis Research Center

NATIONAL AERONAUTICS AND SPACE ADMINISTRATION - WASHINGTON, D. C. - MAY 1970 


\section{ceelno 1. Report No. NASA CR-1595 \\ 4. Title and Subtitle RECIRCULATION PATTERNS IN THE INITIAL REGION OF COAXIAL JETS \\ 2. Government Accession No.}

5. Report Date

- May 1970

6. Porforming Orgonization Codo

7. Author(s) Tzvi Rozenman and Herbert Weinstein a 8. Performing Organization Report No. IIT-CH-E-9

9. Performing Organization Name and Address Dopartment of-Chemical Engineering

$m$.e , Tllinois Institute of Technology Chicago, Illinois

12. Sponsoring Agency Nome and Address

National Aeronautics and Space Administration Washington, D.C. 20546

10. Work Unit No.

11. Contract or Grant No. NGL 14-004-028

13. Type of Repart and Period Covered Contractor Report

14. Sponsoring Agency Code

15. Supplementary Notes

16. Abstract

An experimental study is presented of the initial region in a turbulent coaxial jet flow.

The initial region is the region of the flow upstream of the self-similar flow regime and is strongly influenced by inlet geometry and velocity profile. The investigation is limited to low speed coaxial jets which can be considered incompressible. The outer air stream velocity was held constant at $48 \mathrm{ft} / \mathrm{sec}$ and the inner velocity varied from 0 to $48 \mathrm{ft} / \mathrm{sec}$, to give velocity ratios from infinity to one. The inner stream was either air or Freon 12 which has a density four times that of air. Experimental measurements were made with a hot wire anemometer system and static pressure probes. Shadowgraph pictures were taken to illustrate the flow patterns in the initial region. The data includes average velocity and turbulence intensity in the initial region, and center line and radial distribution of static pressure. The data shows that a back flow region is formed at outer to inner stream velocity ratio of 13 for the homogeneous jet and at outer to inner stream velocity ratio of 26 for the heterogeneous jet. For higher velocity ratio, a circulating toroidal vortex is established which enhances mixing between the inner and outer streams. This vortex pattern exhibits similarity of velocity with velocity ratio variation.

17. Key Words (SugBested by Author(s))

Jets

Initial region

Coaxial

Turbulent flow

Recirculation

19. Security Classif. (of this report) $\checkmark$ Unclassified

20. Security Classif. (of thi s page) Unclassified

8. Distribution Stotement

Unclassified - unlimited

*For sale by the Clearinghouse for Federal Scientific and Technical Information Springfield, Virginia 22151 


\section{FOREWORD}

Research related to advanced nuclear rocket propulsion is described herein. This work was performed under NASA grant NGL 14-004-028 with Mr. Maynard F. Taylor, Nuclear Systems Division, NASA Lewis Research Center, as the Project Manager. The report was originally issued as Illinois Institute of Technology report CH. E. 9. 


\section{TABLE OF CONTENTS}

\section{CHAPTER}

I. INTRODUCTION ...................... 1

II. BACKGROUND ...................... 2

III. THEORETICAL CONSIDERATIONS ............. 8

IV. EXPERIMENTAL INVESTIGATION ............. 12

1. Experimental Apparatus ............. 12

2. Experimental Techniques ............ 17

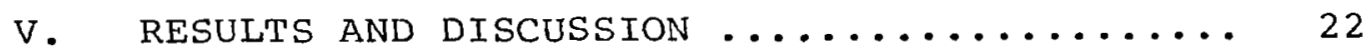

VI. CONCLUSIONS ....................... 68 


\section{NOMENCLATURE}

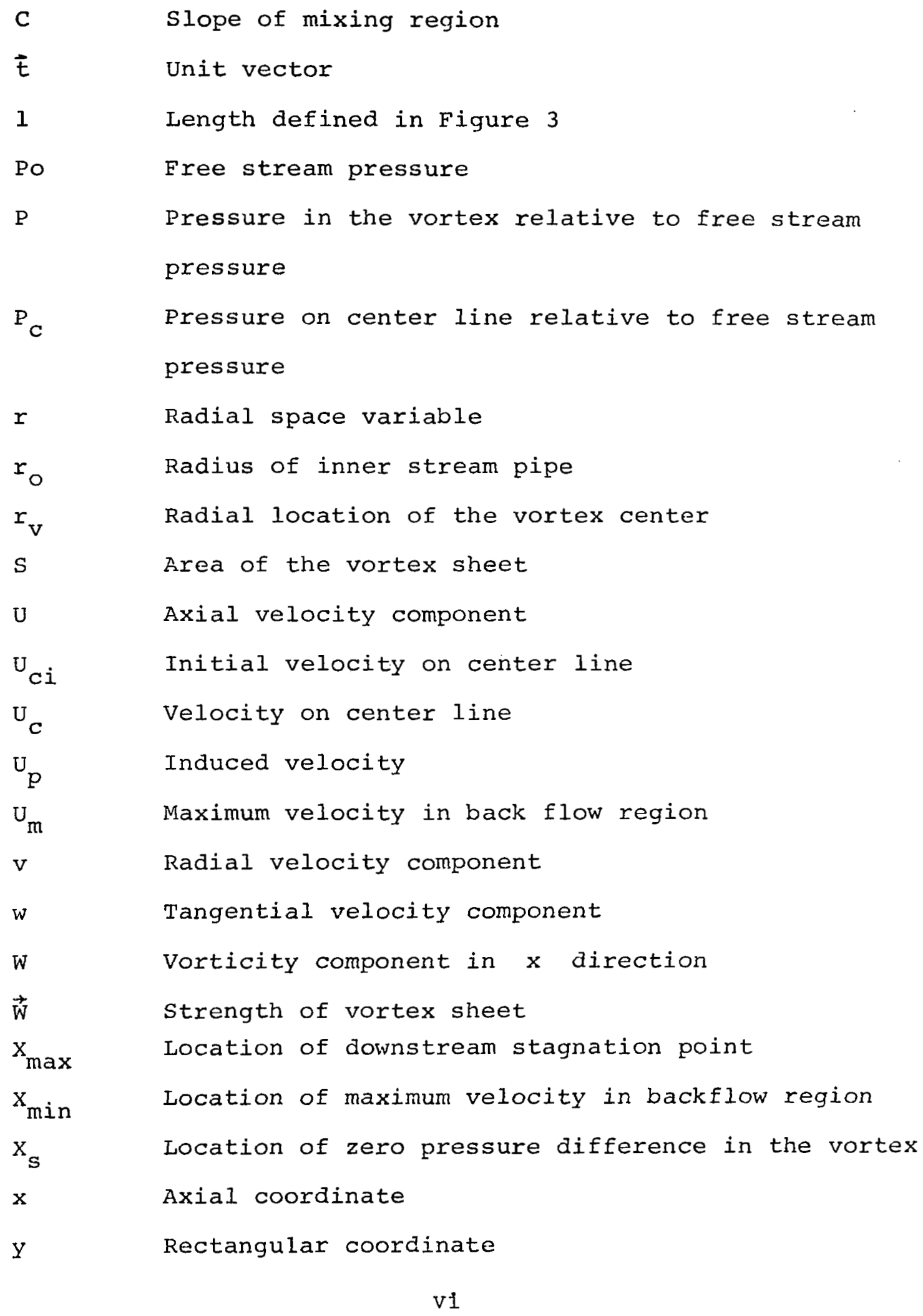


NOMENCLATURE, CONT.

$\begin{array}{ll}\varepsilon & \text { Eddy kinemetic viscosity } \\ \theta & \text { Angle of position vector } \\ \rho & \text { Density } \\ \psi & \text { Angle defined in Figure } 3 \\ \text { Superscripts } \\ \text { - } \quad \text { Time average component } \\ \text { Fluctuating component } \\ \text { Subscripts } \\ \text { c } \\ \text { i }\end{array}$




\section{INTRODUCTION}

The mixing of turbulent coaxial streams of similar and dissimilar fluids is an integral part of many practical engineering problems. Ejectors, afterburners, jet engine combustion chambers, and the concepts advanced for a gaseous core nuclear rocket are only a few examples utilizing a coaxial mixing process.

The coaxial jet is divided into two regions:

a) The initial region.

b) The downstream region.

The downstream region is considered to be the region where, at least experimentally, a similarity exists between velocity profiles at each cross section and a boundary layer type analysis usually fits the experimental data well. The initial region is the region close to the initial cross section of the jet where the mixing region between the jets is established. The flow in this region is usually considered to consist of a potential flow core bounded by an annular mixing region. When the inner stream has a higher velocity than the outer, the velocity remains constant in the potential core and is equal to the initial inner stream velocity. However, very little is known about the initial region of the coaxial jet with faster moving outer stream. As the outer to inner stream velocity ratio is increased, the wake behind the pipe interacts with the issuing inner stream to establish flow patterns which depend on the velocity ratio and the geometry of the system. 
It is the object of this work to investigate these flow patterns.

The results of this work are limited to incompressible low speed coaxial jets with inner stream gas density equal to or greater than the outer stream. The outer stream velocity was kept constant at a value of $U_{0}=48 \mathrm{ft} / \mathrm{sec}$ and the inner stream velocity was varied to give different velocity ratios from $U_{0} / U_{i}=1$ to $U_{o} / U_{i}=\infty$. Two cases were examined:

a) Homogeneous case, where the inner and outer streams were both air.

b) Heterogeneous case, with Freon 12 as the inner jet and air as the outer stream. The density ratio of Freon 12 to air is four.

\section{BACKGROUND}

A schematic diagram of a coaxial jet is given in Figure 1 .

The initial region is the region close to the initial cross section of the jet where the mixing region between the jets becomes established. Kuethe (1), Abramovich (2) and Forstall and Shapiro (3) studied the initial region of an air jet issuing into a coflowing air stream, with the inner jet having a higher velocity than the coflowing stream. The flow in the initial region is considered to consist of a potential core bounded by an annular mixing region. The 


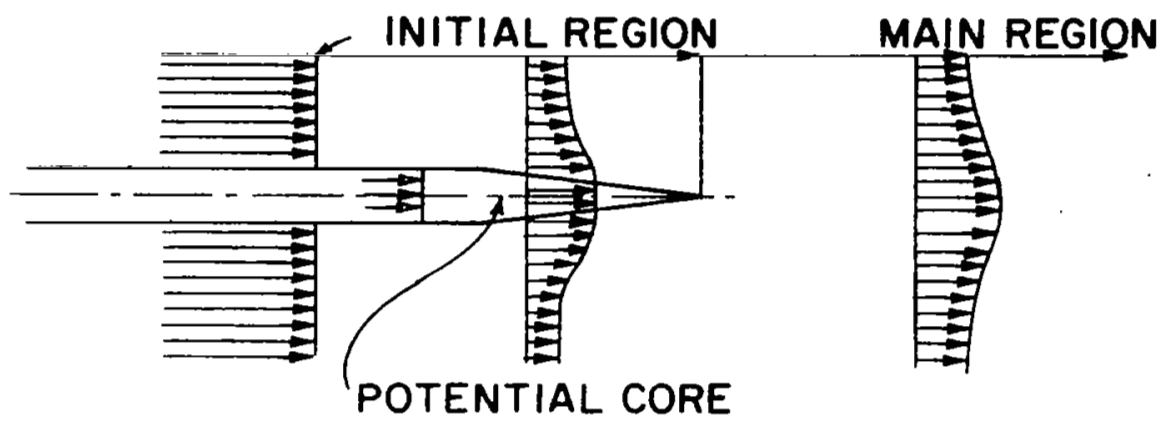

Figure 1. - Schematic diagram of a coaxial jet. 
potential core is considered to be the region where the velocity remains constant and is equal to the initial inner jet velocity. Kuethe found that the length of the potential core was 4.5 nozzle diameters. Forstall \& Shapiro who carried out measurements on a coaxial jet with outer to inner stream velocity ratio of $(.2-.5)$ found that the length of the potential core is determined mainly by the velocity ratio of the two coflowing streams, and presented an empirical relation between length of the potential core and velocity ratio. Abramovich (2) derived theoretical expressions relating the length of the potential core to the initial velocity ratio for two-dimensional and axially symmetric coaxial jets. For a jet issuing into an ambient stream both the expressions of Abramovich and Forstall \& Shapiro reduce to a value of four pipe diameters for the length of the potential core. Hinze and van der Hegge Zijnen (4) give a somewhat higher value of 6 to 8 pipe diameters for the length of the potential core in the submerged jet. Abramovich finds in contrast to Kuethe's work, that the non-dimensional velocity profile in the initial mixing region is universal and does not depend on the velocity ratio. This dimensionless velocity profile coincides with the data of Albertson, et.al. (5) for the initial region of a submerged air jet.

Miller and Comings (6) and Sami, Carmody and Rouse (7) report pressure measurements in the initial region of an air jet issuing into still air. They show that a positive 
static pressure exists within the potential core for a few diameters from the initial cross section, while in the initial mixing region the pressure is negative. These pressure variations are probably a result of the "smoothing" of the viscous velocity profile in the nozzle of the issuing jet.

The downstream region of a coaxial jet is a region where at least experimentally, a similarity exists between the velocity profiles at each cross section. Forstall and Shapiro (3) report a universal non-dimensional velocity profile in the main region of an $(.2-.46)$ outer to inner stream velocity ratio coaxial air jet. This non-dimensional velocity profile is identical to the velocity profile reported by Abramovich for a coaxial jet and also identical to the one obtained by Truppel (8) for a submerged jet.

A universal non-dimensional plot of temperature for an axially symmetric submerged jet is given by Corrsin \& Uberoi and a universal non-dimensional concentration plot is given by Abramovich (2) and Forstall and Shapiro ( 3 ). While the non-dimensional temperature and concentration plots are identical, they are different than the non-dimensional velocity plots. The difference indicates that mass and heat spread faster than momentum.

Until recently, most of the theoretical and experimental works on coaxial jets dealt with the case of the inner jet moving faster than the outer stream. Abramovich shows some theoretical results for the case where the ratio 
of outer to inner stream velocity is $(1-2.5)$. Post of the theoretical results for the initial region agreed with the experimental data. Zawacki and Weinstein (10) carried out extensive measurements of an axially symmetric coaxial jet for which the velocity ratio ranged from 1 to 39.5 with the inner jet having the lower velocity. They also investigated the heterogeneous case, with inner to outer stream density ratio of 4 to 1 and 7 to 1 . Zawacki and Weinstein found that in the downstream region both the velocity and tine concentration profiles can be expressed in a nondimensional form which is independent of the velocity and density ratio. However, the experiments in the initial region show a marked deviation from previously established results for the initial region by other investigators. Substantial velocity variations occur in the potential core, these variations increase with an increase in the velocity ratio. This indicates that the flow characteristics in the initial region of a coaxial jet with high outer to inner stream velocity ratio are different than is predicted by the present jet theories. Abramovich suggests that when the ratio between the outer to inner stream velocity is high, flow reversal of the inner jet may occur near the nozzle exit. In recent years, more investigators have shown interest in this type of flow. A recent paper by Johnson (11) reveals more of the features of the mixing of high velocity ratio coaxial jets. Very little is known about the initial region of the 
coaxial jet with faster moving outer stream. As the outer to inner stream velocity ratio is increased, the wake behind the inner stream pipe interacts with the issuing inner jet to establish flow patterns which depend on the velocity ratio and the geometry of the system.

The existence of an eddy behind a separated boundary layer is typical to wake flow behind solid bodies. Foppel (12) described in 1905 a standing eddy with back flow behind a circular cylinder placed normal to uniform stream. The wake behind a cylinder has been the subject of numerous investigations. The best known of these concern the Von Karman theory of vortex shedding.

Much less work has been done on axisymmetric turbulent wakes behind bluff bodies. Carmody (13) studied the establishment of the wake behind a 6 inch diameter disc placed in a uniform air stream. Carmody's results show the difference between the initial region of a 2-dimensional wake behind a cylinder and the axially symetric wake behind a bluff body. The axially symmetric eddy is more stable, and there exists in it a well defined flow pattern. Calvert (14) studied the shape of the eddy behind cones of different vertex angles placed in a uniform stream. Static pressure, velocity and velocity fluctuations for the different cones were all correlated with a suitable streamwise length scale. Other forms of back flow are reported in the works of Chevray (15) and Chigier and Beer (16).

Their results on jets and wakes can be summarized as 
follows: Both jets and wakes exhibit similarity of average parameters in the downstream region. The initial region of the axially symmetric wake behind bluff bodies consists of a stationary recirculating bubble, whereas the initial region of the coaxial jet with the inner stream of higher velocity than the outer stream displays a constant velocity potential core. The flow patterns in the initial region of a coaxial jet with a higher outer stream velocity have not yet been reported. As the outer to inner stream velocity ratio approaches infinity, the initial region takes the form of the initial region in the wake of a bluff body. However, this wake is different than the one reported by Carmody or Calvert, since no base exists at the upstream initial cross section. The eddy can move into the opening of the inner stream and establish somewhat different flow patterns.

\section{THEORETICAL CONSIDERATIONS}

An analytical description of the inception of recirculation can be obtained from the inviscid theory of vorticity-induced flow. In the vicinity of the initial cross-section of the flow under the end of the inner jet pipe there is a region of very large velocity gradient. This region is made up of the boundary layers on both sides of the inner jet pipe. As these boundary layers separate from the pipe end, they form two interacting 
vortex sheets. The net effect is a rotational region which expands laterally by turbulent diffusion. This rotational region has a. velocity field associated with it. As a limiting case, we will assume that there is no lateral spread of vorticity and the net cylindrical vortex sheet extends to infinity. We will also assume that this vortex sheet is a linear combination of the inner and outer stream vortex sheet as shown in figure 2,

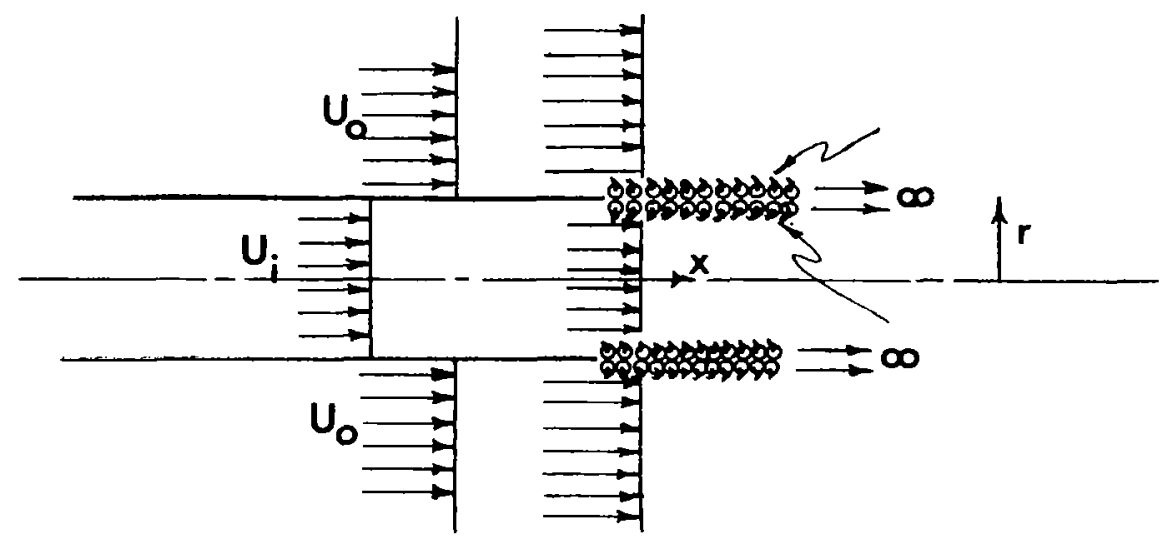

Figure 2. - Idealized vorticity field in a coaxial jet.

The induced velocity field associated with a vortex sheet can be expressed as follows (17):

$$
\overrightarrow{\mathrm{U}}_{\mathrm{p}}=\frac{1}{4 \pi} \int_{(\mathrm{s})} \frac{\overrightarrow{\mathrm{W}} \times \overrightarrow{\mathrm{r}}}{\mathrm{r}^{3}} \mathrm{ds}
$$


where,

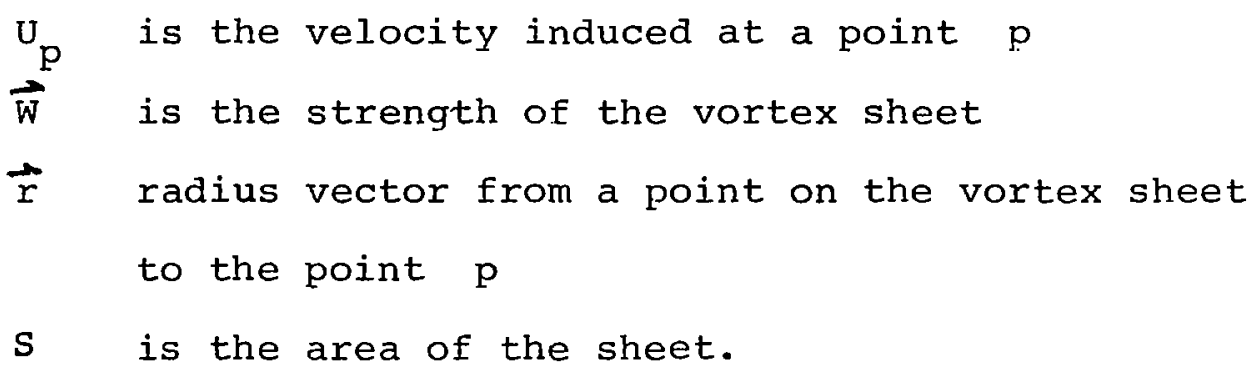

We wish to find the induced velocity at the center of the initial cross section. The system of coordinates is given in figure 3 .

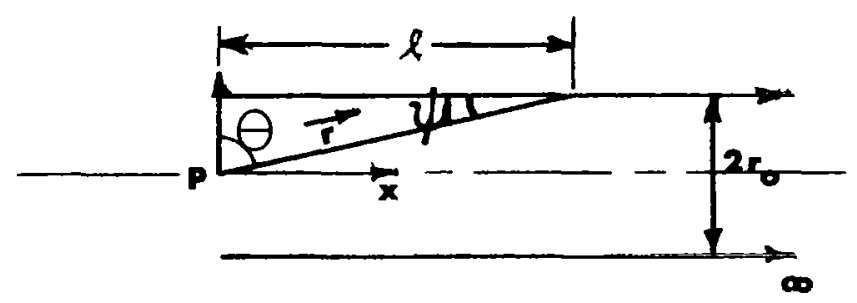

Figure 3. - The system of coordinates.

The strength of the outer vortex sheet is $U_{0}$ directed in the ${ }^{+i_{\theta}}$ direction. The inner sheet has a strength $U_{i}$ and is directed in the opposite direction $-i_{\theta}$.

Thus,

$$
\vec{W}=\left(U_{0}-U_{i}\right) \vec{i}_{\theta}
$$

and,

$$
\vec{r}=-\left(\vec{r}_{0}+\vec{l}\right)=-r_{0} \vec{i}_{r}-\ell \cdot \vec{i}_{x}
$$

Now,

$$
r=\frac{r_{0}}{\sin \psi}, \quad l=\frac{r_{0}}{\tan \psi}, \quad d s=2 \pi r_{0} d \ell
$$

Substituting, 


$$
\mathrm{ds}=-2 \pi r_{0}^{2} \frac{\mathrm{d} \psi}{\sin ^{2} \psi}
$$

and,

$$
\vec{w} \times \vec{r}=-\vec{i}_{r} \ell\left(U_{0}-U_{i}\right)+\vec{i}_{x} r_{0}\left(U_{0}-U_{i}\right)
$$

Thus the induced velocity at the point $p$ is,

$$
U_{p}=\frac{1}{4 \pi} \int_{(S)} \frac{\vec{i}_{x} r_{0}\left(U_{0}-U_{i}\right)-\vec{i}_{r} \ell\left(U_{0}-U_{i}\right)}{r_{0}{ }^{3}}\left(-2 \pi r_{0}^{2} \sin \psi d \psi\right)
$$

The $\mathrm{x}$ component of $\mathrm{U}_{\mathrm{p}}$ is,

$$
\mathrm{U}_{\mathrm{p}}=-\frac{\mathrm{U}_{0}-\mathrm{U}_{\mathrm{i}}}{2} \int_{0}^{\pi / 2} \sin \psi \mathrm{d} \psi=-\frac{\mathrm{U}_{0}-\mathrm{U}_{\mathrm{i}}}{2}
$$

The induced velocity is directed inward into the pipe and its value is $\frac{\mathrm{U}_{0}-U_{i}}{2}$. Since we assurne that the velocity of the inner jet is $U_{i}$, back flow will occur when $\mathrm{U}_{0}=3 \mathrm{U}_{i}$. This idealized picture of the vorticity interaction in the initial region of a coaxial jet indicates that if the velocity ratio $\mathrm{U}_{0} / \mathrm{U}_{i}$ is greater than 3 a recirculating eddy will be formed. This is only a limiting case. However, the analysis indicates that back flow is possible due to the large vorticity of the separated outer stream. The fact that velocity level does not appear in the solution indicates that it only has a second order effect in determining when recirculation starts in a real turbulent flow. Recently, Shavit and Lavan (18) solved the complete vorticity equation for laminar coaxial mixing, and 
report the calculation shows an eddy at the initial region of the jet.

IV. EXPERIMENTAL INVESTIGATION

\section{Experimental Apparatus}

The apparatus used in obtaining data for the coaxial flow system was the same one that was used by Zawacki and Weinstein (10). See figure 4. A vertical column that had a square cross section of 8 inches and constructed of $3 / 4$ inches thick plexiglass was divided into three sections. The first was a 24 inch entrance region which was long enough to provide a parallel flow field without too much boundary layer buildup. The second section was a 36 inch long test section where mixing of the two streams took place. The third section was a short 12 inch section filled with card board honeycomb to prevent any swirling of the fluid due to exit effects. The bottom of the test section was connected to the inlet of the blower with 8 inch diameter sheet metal tubing. A Buffalo forge type GE, low pressure, high capacity blower was used to provide the suction for the outer stream of the system. The inner stream was introduced into the center of the column by a four foot long stainless steel tube, with an outer diameter of $3 / 4$ inches and a wall thickness of 0.0135 inches. The velocity profile in the inner pipe was fully developed. 


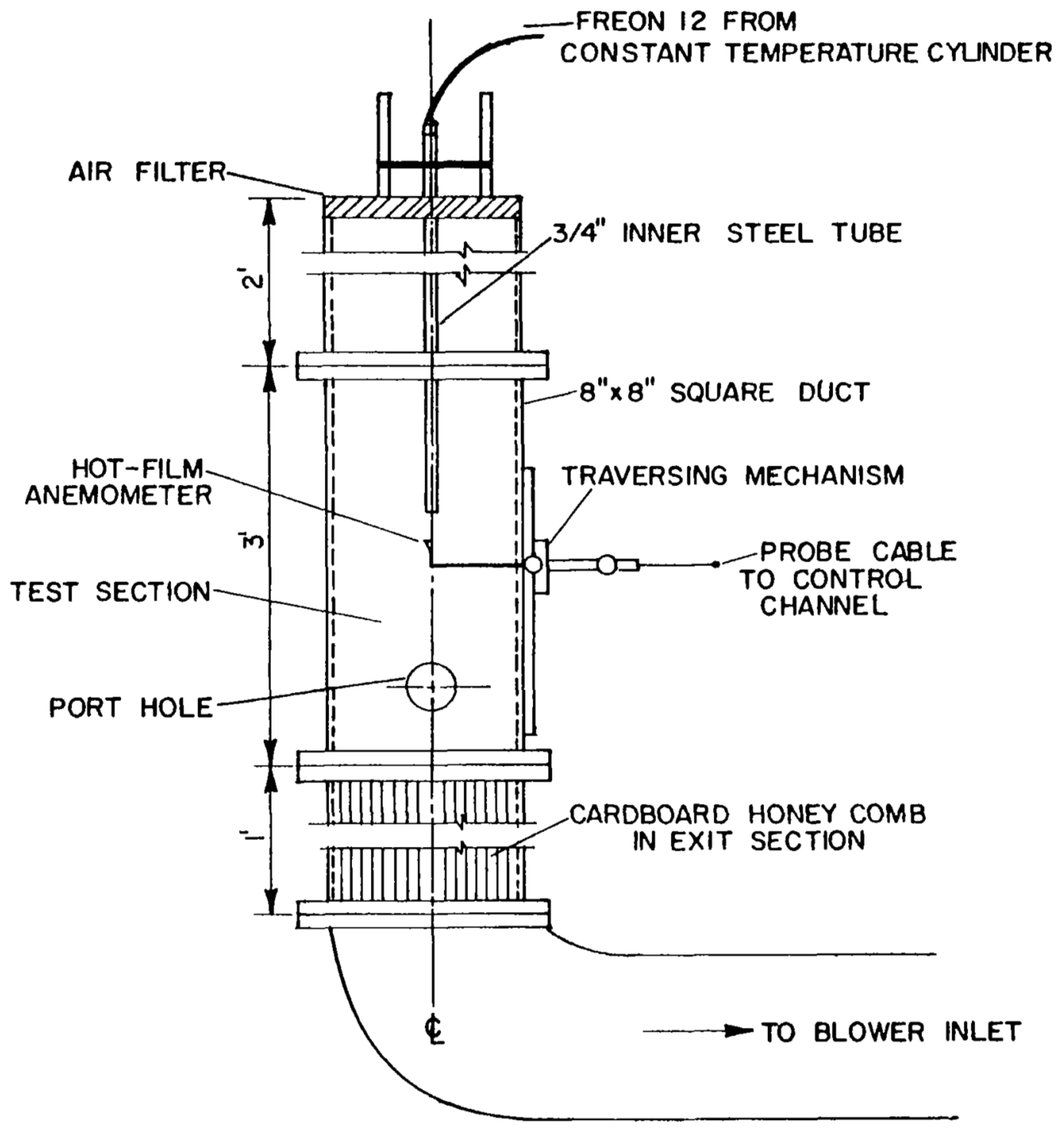

Figure 4. - Experimental apparatus. 
A traversing mechanism, with both lateral and axial movement, was mounted on the 36 inch test section of the column. A 24 inch slot was milled through the mechanism and the plexiglass to provide access to any point in the mixing region.

Three high precision Brooks rotameters were used to measure the volumetric flow of air and Freon to the inner stream. The Freon was supplied from 145 pound cylinders. The hot film anemometry instrumentation needed for measurement of velocity, concentration and turbulence quantities were supplied by thermo systems incorporated of Minneapolis, Minnesota. Two independent channels were provided to control two films at a constant temperature. Each unit was equipped with a linearizer that provided a direct measure of the power dissipation of the film. A HewlettPackard root mean square voltmeter was supplied to measure the r.m.s. voltage of each signal. A Tektronix $502 \mathrm{~A}$ dual beam oscilloscope was used to monitor the outputs of the controlling channels. Two types of probes were supplied with the system. The first was a single hot film, 0.002 inches in diameter and .04 inches in length. The second type of probe was the aspirating probe, which was a .001 inch diameter hot film mounted in a .08 inch inner diameter tube. A jewel bearing, with a $0.007-0.009$ inch diameter hole drilled in it, was mounted in the tube in back of the wire. A Cenco vacuum pump was used to withdraw the sample from the system, past the hot film and through the bearing. The 
vacuum pump provided sufficient pressure drop so that there was sonic flow through the jewel bearing at all times.

Two types of pressure probes were used in the experiment. A static pressure probe was constructed to measure the ambient pressure in the mixing region. The probe was made of a .032" stainless steel hypodermic tube with side holes of .015" in diameter. Since a slight vacuum of approximately 4 inches of water existed in the test apparatus due to the suction of the blower, another reference probe was used with the measuring probe as shown in figure 5a. The reference probe was similar to the measuring probe, but was held in the outer stream at a distance of 6 inner pipe radii from the measuring probe. It was assumed that the reference probe monitored the free stream static pressure. This assumption has been checked and verified in the experiment.

The second probe consisted of a long cylindrical hypodermic tube of .064" O.D. Two holes of .008" in diameter, were drilled on the surface of a short brass cylinder with azimuthal angle of $80^{\circ}$ (Figure 5b). The holes were connected to the hypodermic tube by similar holes drilled in the brass cylinder parallel to its axis. Each hole was connected to an opposite section of the tubing. The two edges of the hypodermic tube were connected to the two sides of a differential micromanometer that measured the difference in pressure of the azimuthal holes. This probe was used to find direction of the flow, and is the 


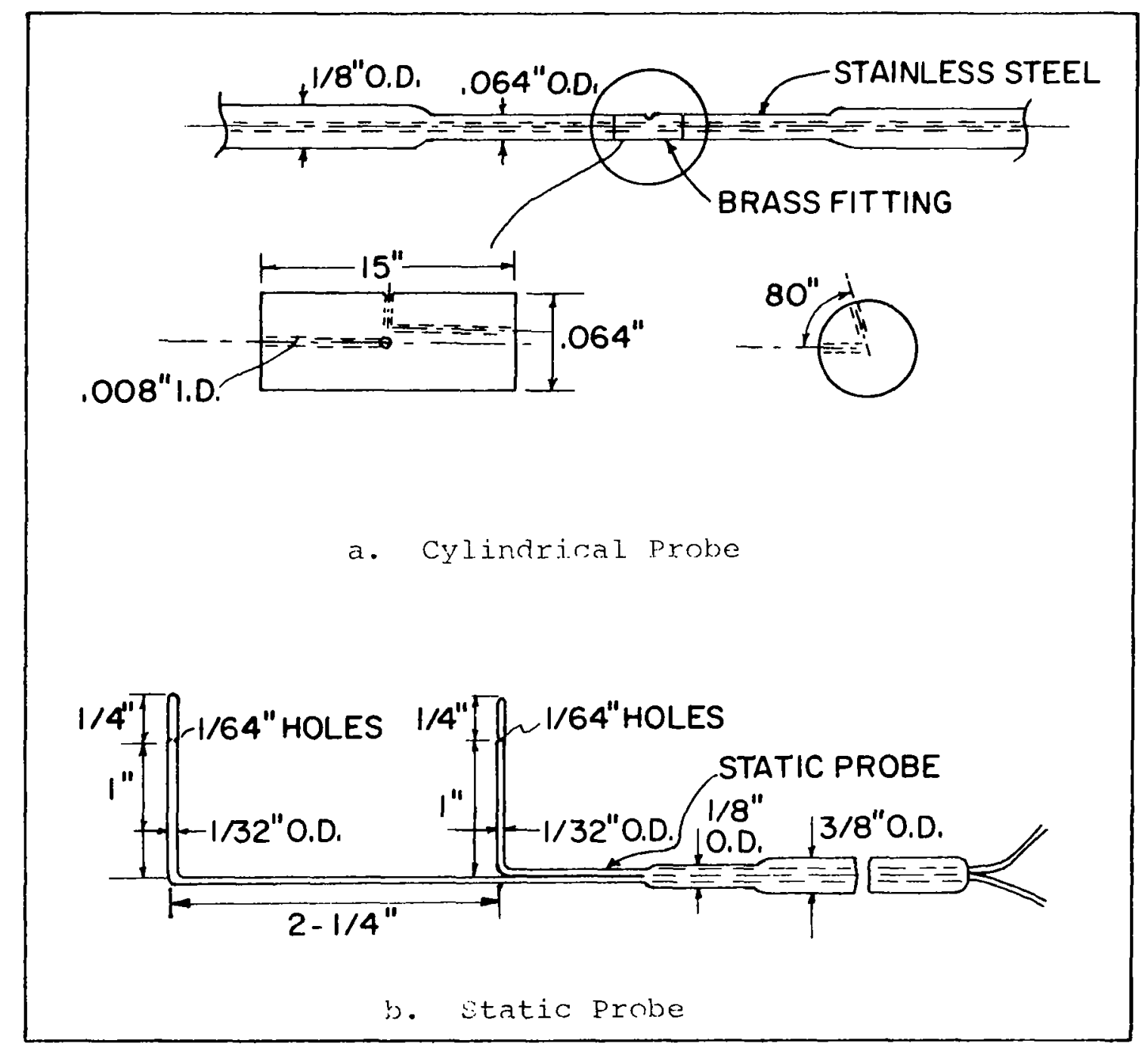

Figure 5. - Details of pressure probes. 
same probe that was used by Lavan and Fejer (19).

\section{Experimental Techniques}

For measurement of average velocities and turbulence intensities, a single hot film was used. The probe was mounted in a horizontal position directly connected to the probe holder without using any adaptor. Setting the probe in this position minimized the interference caused by any adaptor with the flow patterns in the initial region. The measurements were taken in the region between the mouth of the jet and 3 inches downstream, which is equivalent to $x=8.5 r_{0}$.

Since backflow was expected to exist in the initial region and because the hot film does not respond to the direction of flow, the sign of the measured velocity had to be determined separately. For high velocity ratios, where back flow was suspected to take place, a center line velocity profile as shown in figure 6 a was recorded with the film. The corresponding true velocity is shown in figure 6b. The minimum points in figure 6 a correspond to zero velocities and hence to stagnation points in the true velocity profile.

Stagnation points were located with the cylindrical probe. The downstream stagnation point was located as follows: The probe was placed at an axial distance of 2 inches. The center stream line direction was determined first by finding the azimuthal angle for which the pressure 


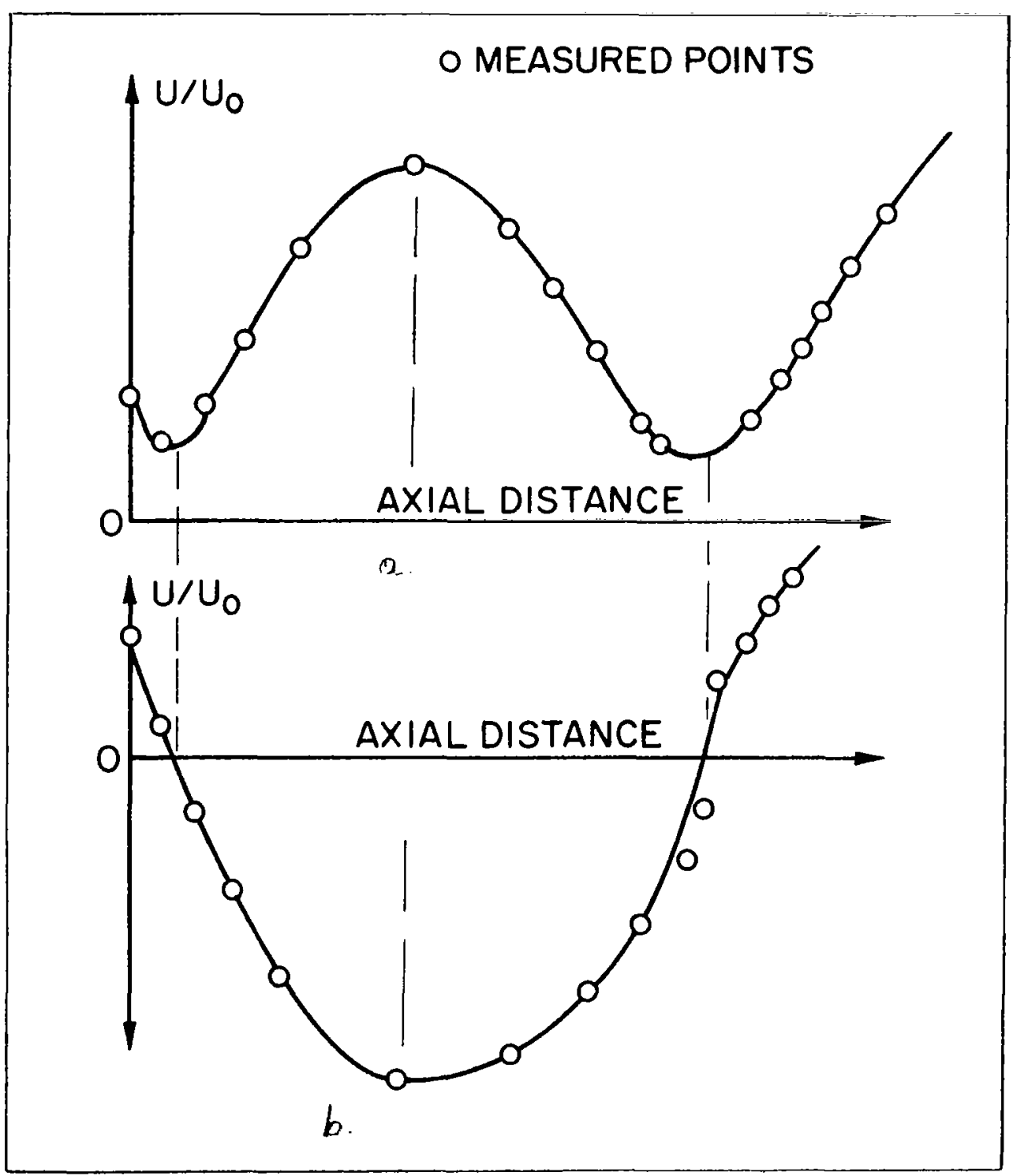

Figure 6. - Comparison between wire reading and true velocity profile. 
difference between the holes was zero, and then the probe was rotated $40^{\circ}$ - half the angle between the holes. At this position, one of the holes was facing upward with axis coinciding with the center line. The probe was then slowly moved in the unstream direction and the pressure difference between the two holes was constantly monitored. A point was reached where the pressure difference was zero. The reading remained zero even when the probe was rotated in all azimuthal directions. This point was assumed to be the stagnation point.

The vortex center was similarly found. The probe was moved in a radial direction at different cross sections. A point was reached where the pressure difference was nearly zero and remained practically constant when the probe was rotated in different azimuthal directions. It was impossible to locate the upstream stagnation point this way. Zero value pressure differences were not recorded in the region close to the initial cross section.

The location of the stagnation point and vortex center agreed well with the location of zero velocity found by the hot wire.

The pressure was measured with an inclined manometer. The range of the manometer was from zero to .02 inches of water with reading accuracy of $5 \times 10^{-4}$ inches. Pressures were taken of the center line for different cross sections. The axial and radial distance were measured with a precision ruler of $1 / 100$ inches in accuracy. 
According to Tmoore (20) when a static probe is aligned with the direction of mean flow the error in the pressure reading due to turbulence is $\pm \frac{1}{2}\left(\overline{v^{\prime 2}}+\overline{w^{\prime 2}}\right)$. No agreement could be reached on the sign of the correction. It seems that turbulence affects the pressure reading in two ways. The turbulence intensities together with the main flow will combine to give an effective velocity direction which is different than the mean flow direction. Since the probe is aligned with the direction of the mean flow the pressure reading will be reduced. On the other hand, the lateral turbulence intensities exert an impact back on the holes, thus increasing the pressure reading. These two effects will depend on the type of turbulence in the flow. If the scale of turbulence is small relative to probe diameter as in the flow behind a grid, the sign of the correction term $\frac{1}{2}\left(\overline{\mathrm{v}^{\prime 2}}+\overline{\mathrm{w}^{\prime 2}}\right)$ is positive. The sign will be negative in a flow of which the turbulence scale is large compared to probe diameter. It is hard therefore to correct the pressure measurements for turbulent effects. Miller and Comings ( 6 ) who measured static pressure with a disc type probe and Chevray (15) who measured static pressure in the wake behind a body of revolution using a static probe, did not correct their pressure reading for the effect of turbulence. In any event, the resulting correction in the present work would not have been more than $2 \%$ of the dynamic head of the outer stream. 
Flow visualization techniques were employed to obtain more insight into the mixing process. The mixing process of the Freon and air stxeams establishes regions of changing density. These regions can be recorded on a photographic plate using the shadowgraph technique. The shadowgraph picture depends on the second derivative of density, and hence quantitative analysis of the picture is extremely difficult. However, since the mixing process in the present case is of a turbulent nature, a qualitative analysis of the details of the flow is possible.

The shadowgraph pictures were taken with a 1530 GR single flash strobolum. The flash was of 1 second time duration and its intensity was 5 million candles. Two types of photographic film were used:

a) A 3000 ASA $51 / 2 " \times 41 / 2 "$ polaroid film

b) A 1600 ASA 10" x 8" Kodak film

The polaroid was mainly used for investigating the initial region while the negative film was used to photograph the entire jet including the main region. The negatives were printed on high contrast Agfa paper.

At the actual picture taking, the strobolum was placed at approximately 12 feet from the jet. The flashing lamp was covered with aluminum foil and a small hole $1 \mathrm{~mm}$. in diameter was made at the center to achieve better resolution. The photographic film was placed in a plate with a movable screen.

The plate was placed at about 10 inches from the jet. 
The room was blackened and the light was turned off. The screen was removed from the plate and the strobolum was triggered to cause the flash. Following the flash the plate was closed and the light was turned on. The film was later developed. This procedure was repeated for all the velocity ratios used.

In the homogeneous jet the back flow region was studied with a Freon tracer injection technique. Small quantities of Freon $12(20 \mathrm{cc} / \mathrm{min})$ were injected through a .064" diameter hypodermic tube either at the center line of the inner jet or half-way between the center and inner jet wall. The issuing velocity of the tracer was adjusted to be a little greater than the center line velocity of the undisturbed velocity profile of the inner jet. The pattern of the Freon tracer is visible on the shadowgraph picture and indicates the axial stream line direction.

\section{RESULTS AND DISCUSSION}

The first series of experiments was carried out in order to determine the effect of the initial velocity ratio on the ratio of center line velocity to initial center line velocity and the axial turbulence intensity through the initial region. The velocity of the outer jet was kept constant at a value of $U_{0}=48 \mathrm{ft} / \mathrm{sec}$ and the average velocity of the inner jet was varied to give the ảifferent velocity ratios. The 
turbulent intensity of the outer stream was about 38 . Figure 7 shows that the center line velocity remains at a constant value for a short distance, decreases, reaches a minimum and then increases. As the velocity ratio is increased, the point of minimum velocity moves closer to the jet initial cross section. The turbulence intensities in figure 8 increase in magnitude from the initial face, reach a maximum and decrease further downstream. The velocities are normalized on the initial face centerline velocity with no outer flow.

These results are different from the results for the initial region of the jet which were reported by Forstall and Shapiro (1) or Abramovich (2). In the present case of a high outer to inner stream velocity ratio coaxial jet, the length of the "potential" core is smaller than for a submerged jet or low velocity ratio coaxial jets. Furthermore, for velocity ratios greater than 3.5 , the constant velocity potential core practically disappears and a constant velocity region does not exist.

Figures 9 and 10 show the change in center line velocity and concentration of the inner jet for different velocity ratios in the heterogeneous case. Four velocity ratios were used, $\frac{U_{0}}{U_{i}}=4,6,12,20$. The behavior in the heterogeneous cases is similar to that of the homogeneous cases. The velocity decreases, reaches a minimum and then increases. In the heterogeneous case, the potential core region is longer than in the homogeneous case and disappears 


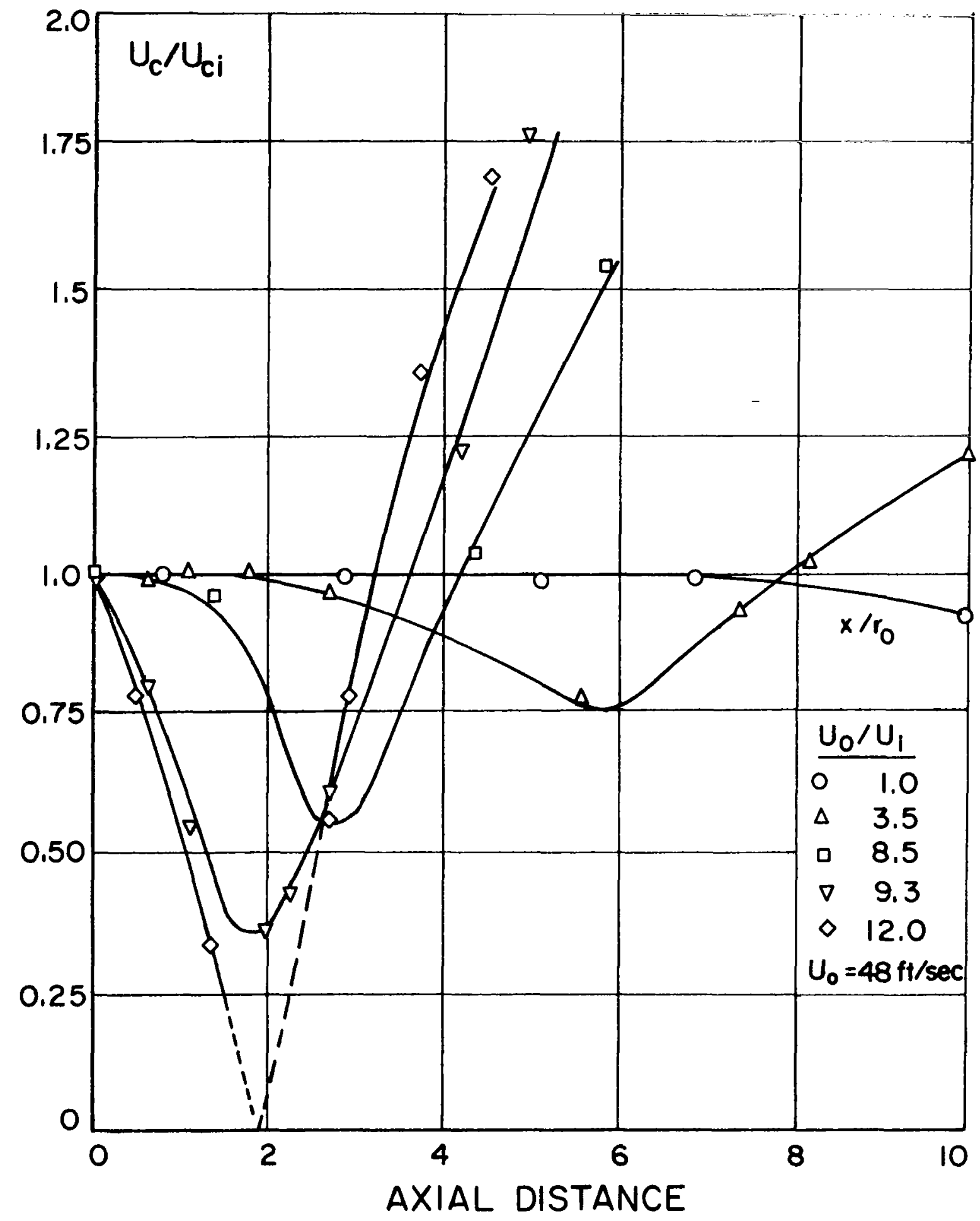

Figure 7. - Decay of centerline velocity as a function of initial velocity ratio, homogeneous jet. 


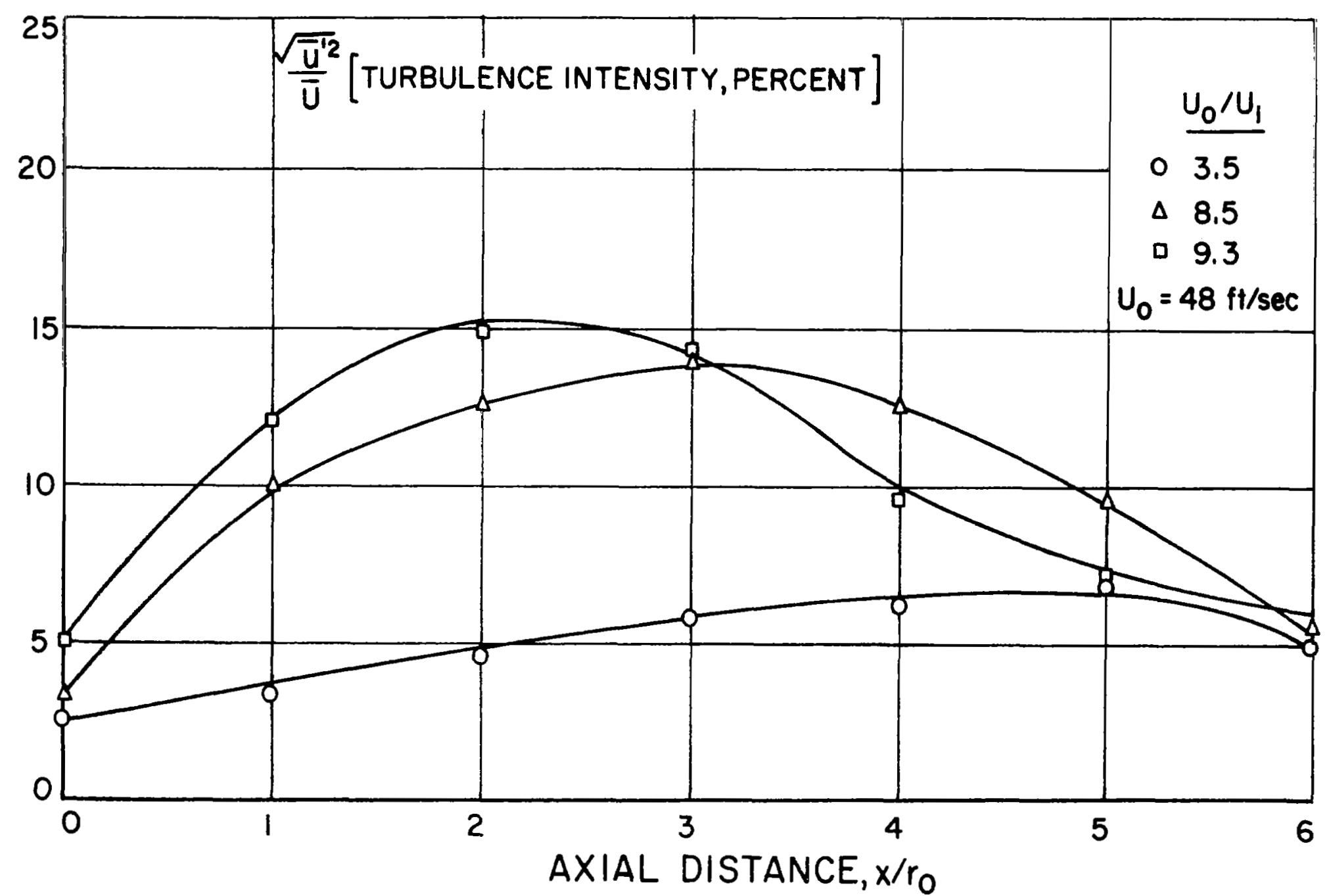

Figure 8. - Centerline turbulence intensity as a function of axial distance, homogeneous jet. 


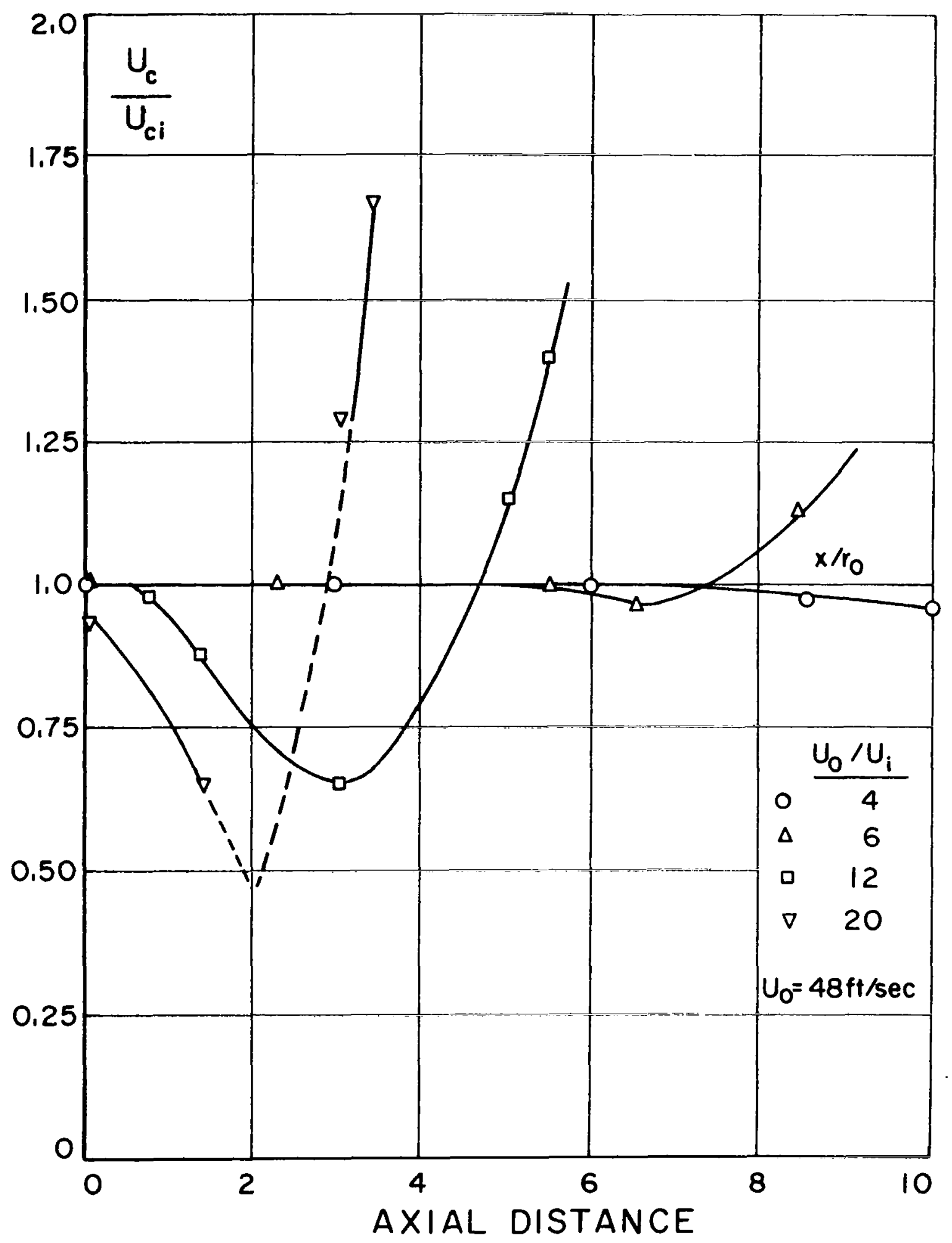

Figure 9. - Decay of centerline velocity as a function of initial velocity ratio, heterogeneous jet. 


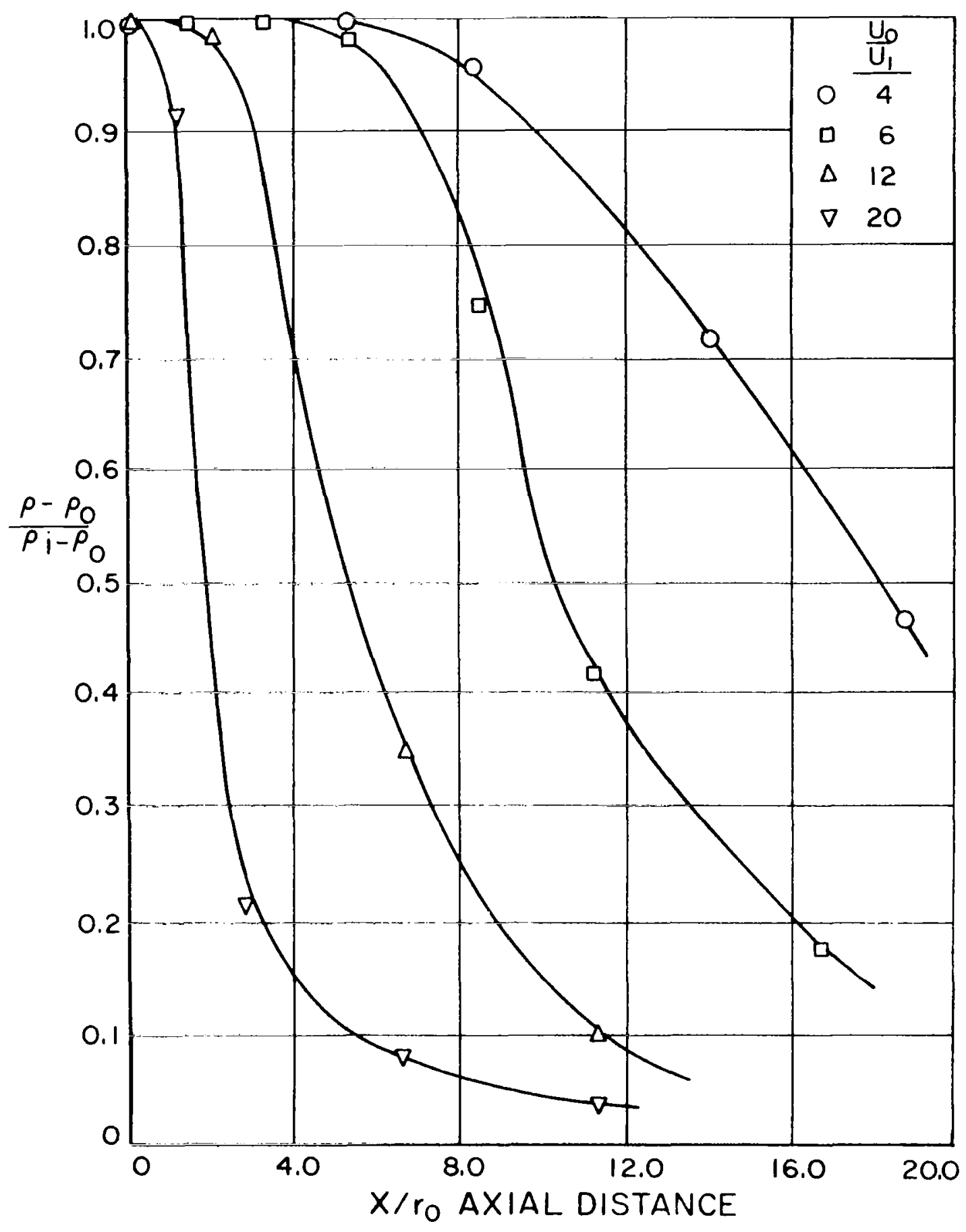

Figure 10. - Decay of centerline density as a function of initial velocity ratio. 
at a higher velocity ratio of approximately 14. This is attributed to increase in the momentum of the inner stream by a factor of 4 . In figure 10, the length of the constant density potential core is approximately equal to the length of the constant velocity core. However, a sharp decrease in concentration is evident for the high velocity ratio cases. For $\frac{\mathrm{U}_{0}}{\mathrm{U}_{i}}=20$, the nondimensional concentration decays to 1/10 of its initial value in four radii. Zawacki and Weinstein (10), who measured concentration profiles far downstream, report a similarity region for the concentration starting at $\frac{x}{r_{0}}=11.2$. Thus more than $90 \%$ of the center 1 ine concentration decay takes place upstream of the similarity region.

From figures 7 and 9 it is evident that after reaching a minimum, the low velocity inner jet (high velocity ratio) is accelerated faster than the high velocity inner jet (low velocity ratio). The center line velocity of an initially low velocity jet exceeds the center line velocity of a higher velocity jet.

This behavior in the initial region, of decreasing and then increasing center line velocity, is typical of a high velocity ratio coaxial jet system when the outer stream is faster than the inner stream. Figure 7 shows that as the velocity ratio is increased, the minimum in the velocity profile is lower. If the velocity ratio is further increased, the minimum velocity can reach zero values or even become negative. Thus, as the velocity 
ratio is increased further, a flow reversal region will be established. When the outer faster stream separates from the outer boundaries of the inner jet pipe it starts to entrain fluid from the central region. This entrainment is due to the shearing action of the faster moving jet. Through the process of entrainment of the outer part of the slower jet, the stream lines in the region close to the outer jet will converge since the fluid is accelerated. Since the mass flow of the inner jet is constant, the stream lines close to the center line will diverge as a result of the deceleration of the fluid in order to satisfy the continuity condition. Thus, a region of positive pressure is generated on the center line of the jet. The inner jet is then subjected to two forces. The faster annular stream accelerates the inner jet and the downstream positive pressure tends to offset this force and retard the inner fluid. If the pressure rise exceeds the dynamic head at the center line of the inner jet, a complete reversal of the flow will occur with the formation of a closed recirculation region. If, however, the momentum of the inner stream is high enough, the accelerated outer region will reach the center line before a complete reversal occurs. At this point the velocity of the center line reaches its lowest value, the inner jet is entirely absorbed by the outer stream, the pressure gradient drops further downstream and the whole flow behaves as a wake does.

Figure 11 shows the possible configuration of the flow 


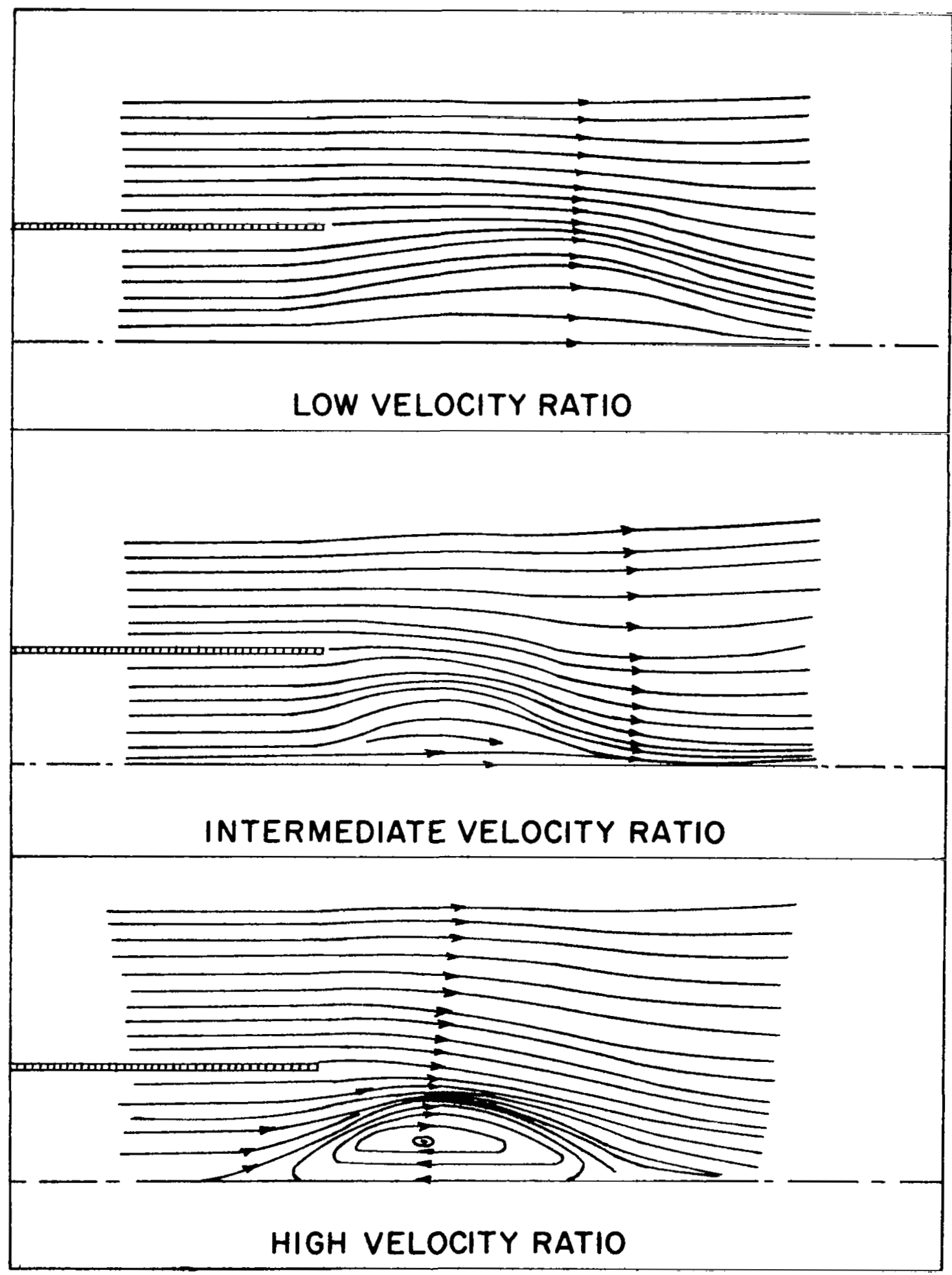

Figure ll. - Effect of velocity ratio on the stream lines in the initial region of the coaxial jet. 
patterns. For low velocity ratio, the stream lines are slightly diverted from their original direction. For intermediate velocity ratios, there will be an appreciable decrease in the center line velocity. The stream lines converge at the interface between the two jets and diverge at the center line. In both cases the laterally expanding rotational region at the interface between the jets induces potentially the diverging stream lines near the center line. For high velocity ratio, complete flow reversal occurs. All the initial inner stream lines are diverted from the center line. A toroidal vortex is generated with forward and backward stagnation points and a ring vortex center.

A generalization for the definition of the potential core in the initial region of a jet is needed to include the observations of the present work. A potential core still exists in the coaxial jet under consideration. However, this potential core is not simply specified by a constant velocity. It is a potential flow region of varying velocity. The velocity in the potential core essentially changes isentropically and viscous or turbulent energy losses are negligible. Then by applying Bernoulli's theorem on the center stream line, the potential core length is defined as the length over which,

$$
1 / 2 \mathrm{U}^{2} \mathrm{ci}+\frac{\mathrm{p}_{\mathrm{Ci}}}{\rho}=\text { constant. }
$$

$\mathrm{U}_{\mathrm{Ci}}$ and $\mathrm{P}_{\mathrm{Ci}}$ are the initial values of the center line 
velocity and pressure of the inner jet.

When back flow occurs in the initial region the upstream stagnation point pressure $\mathrm{p}_{\mathrm{s}}$ is expressed as:

$$
\frac{p_{s}-p_{i}}{\rho} \approx 1 / 2 \mathrm{u}_{c i}^{2}
$$

This definition of the potential core in the initial region is more general than the constant velocity potential core definition.

The shadowgraph pictures of the coaxial jet give more insight into the mixing process. Plates 1, 2, 3, 4 show an inner Freon 12 jet mixing with a coflowing air stream at velocity ratios of $\frac{U_{0}}{U_{i}}=2,4,10,20$. The shadowgraph picture is a two-dimensional projection of a three dimensional phenomenon. The shadows on the plates are an integrated result of the variation in the intensity of a light beam passing through the jet. However, the edge of the jet can be considered as two dimensional because of its small thickness. Hence, the intermittency in the shadows on the picture can be assumed to represent the intermittency of the turbulence in the boundary layer of the jet. It is also evident that varying velocity ratio has a small effect on the spreading of the jet. In all four shadowgraphs, the width of the visible jet as a function of axial distance remains almost the same for the four velocity ratios in the experiment. The boundaries of the jet follow approximately a straight line with a slope of $\mathrm{C}=.05$. Abramovich 


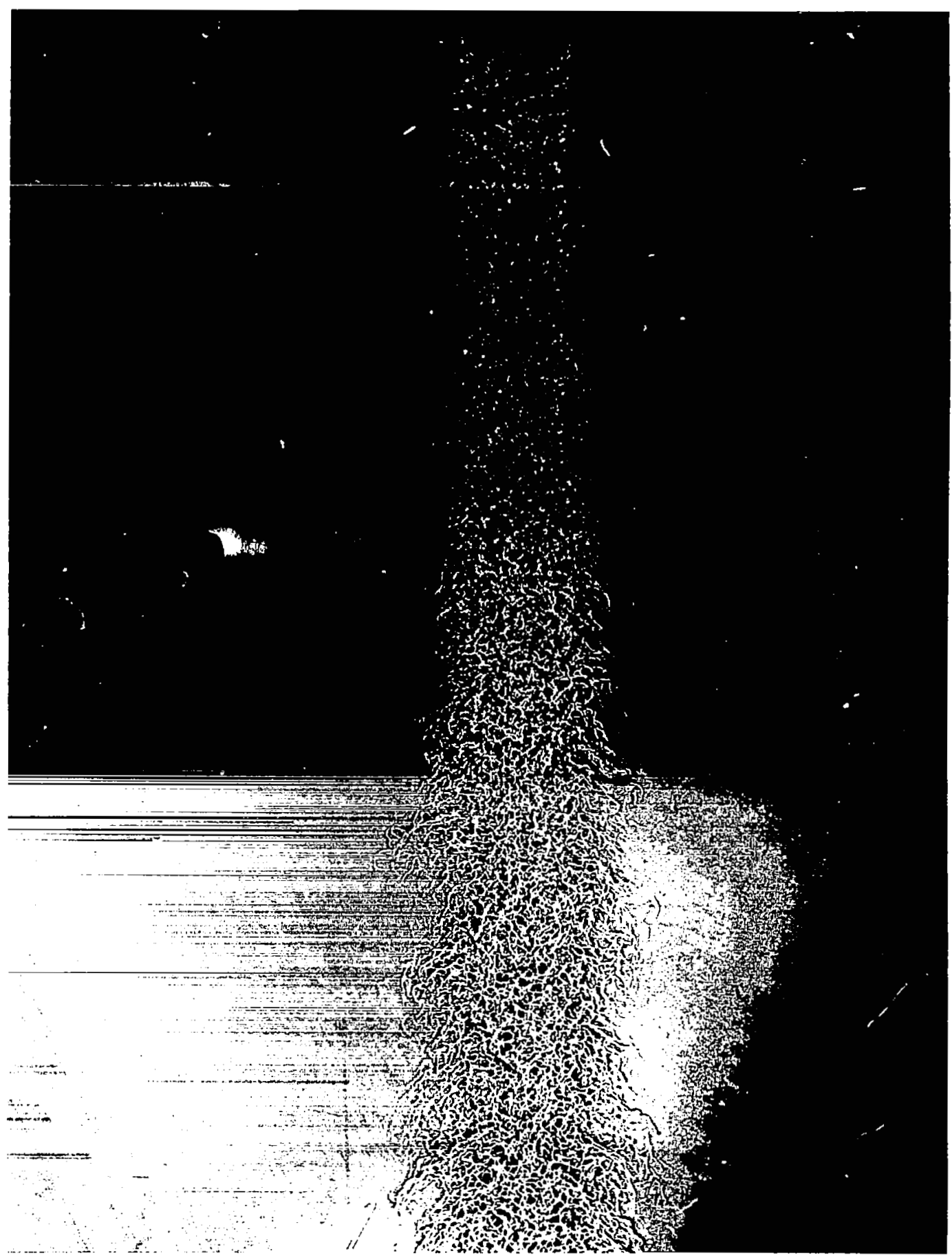

Velocity ratio:

Freon jet issuing into moving air stream

$$
\frac{u_{0}}{u_{i}}=2
$$$$
\text { Plate } 1
$$ 


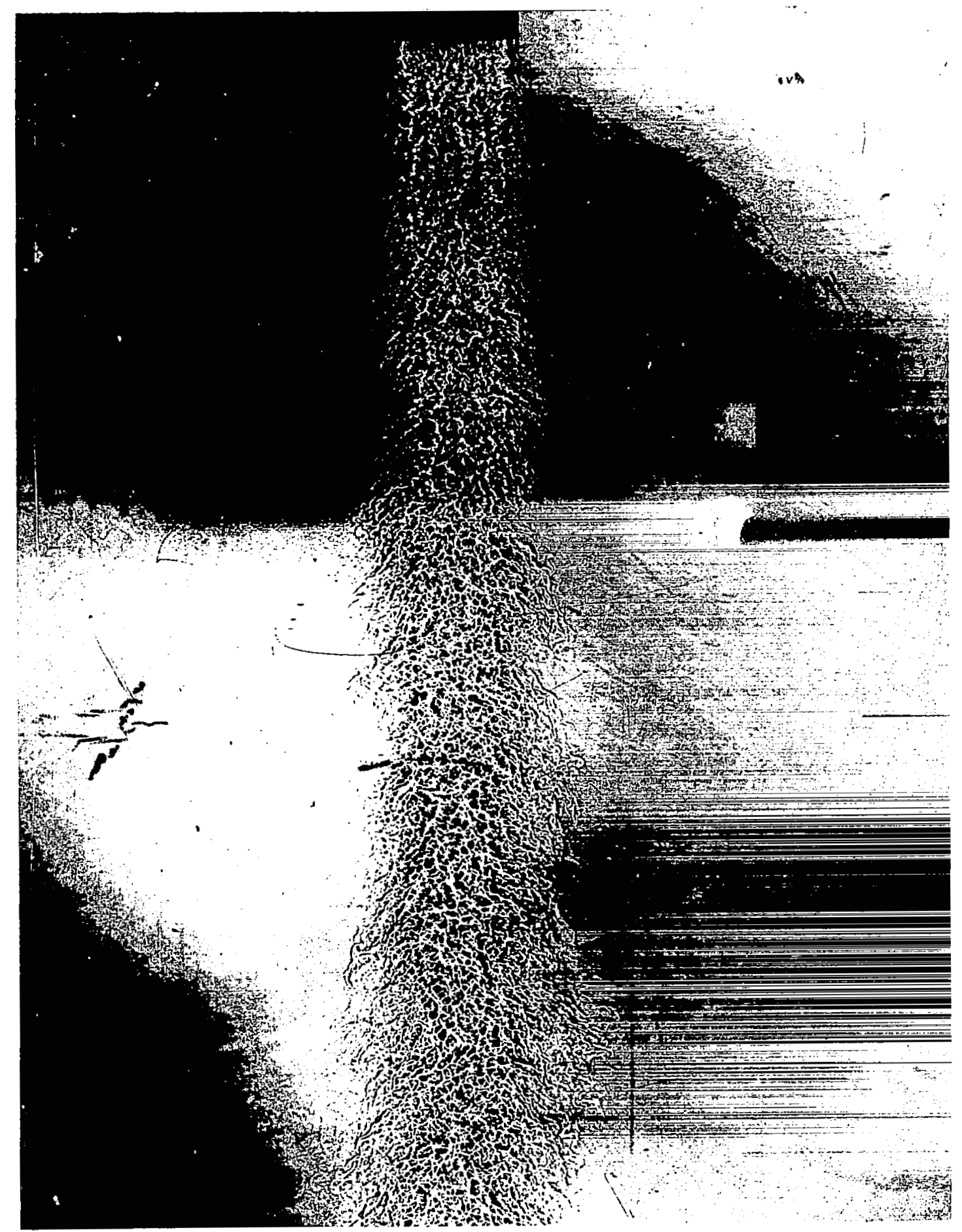

Freon jet issuing into moving air stream

Plate 2
Velocity ratio:

$$
\frac{u_{0}}{u_{i}}=4
$$




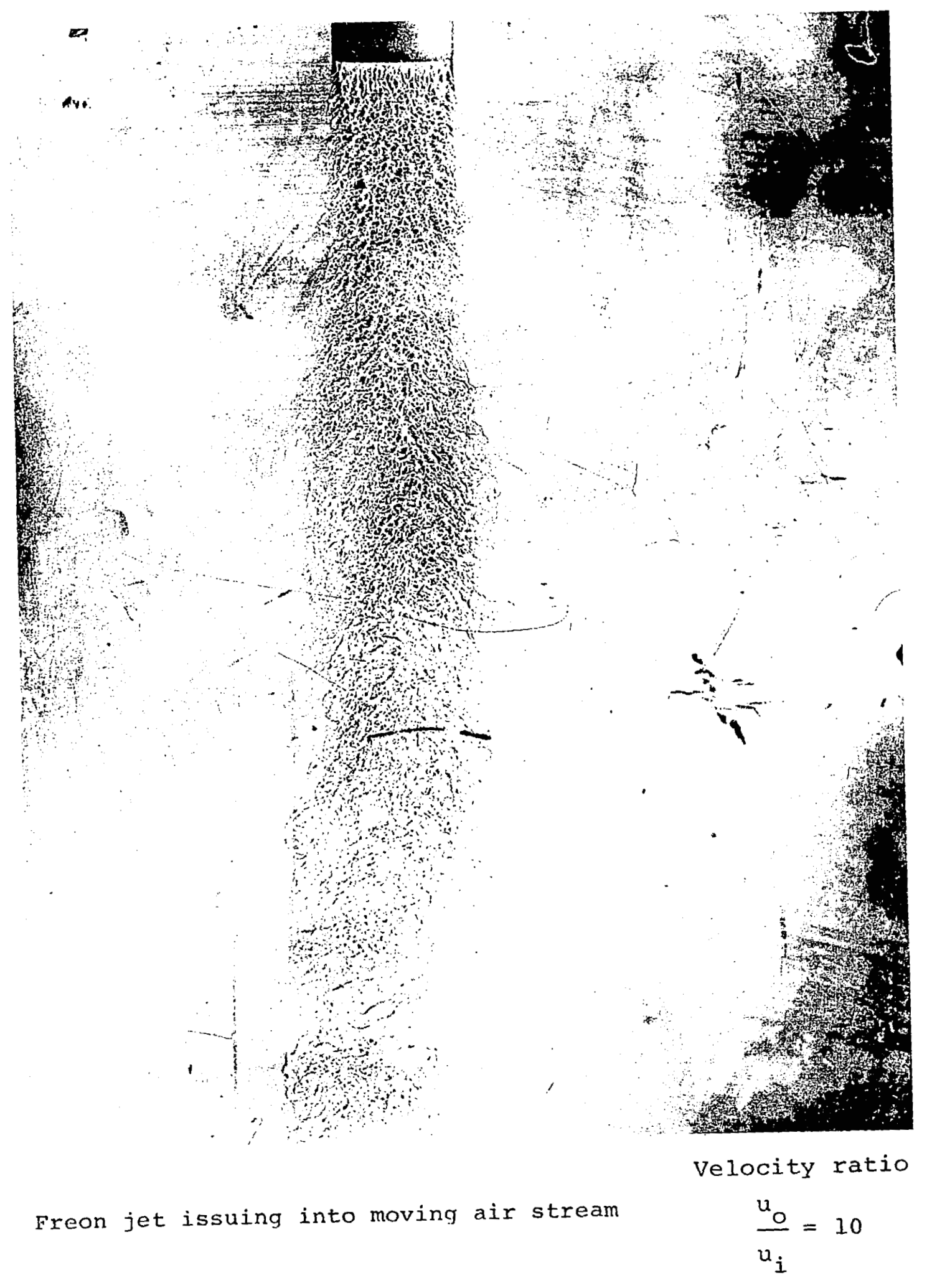

Plate 3 


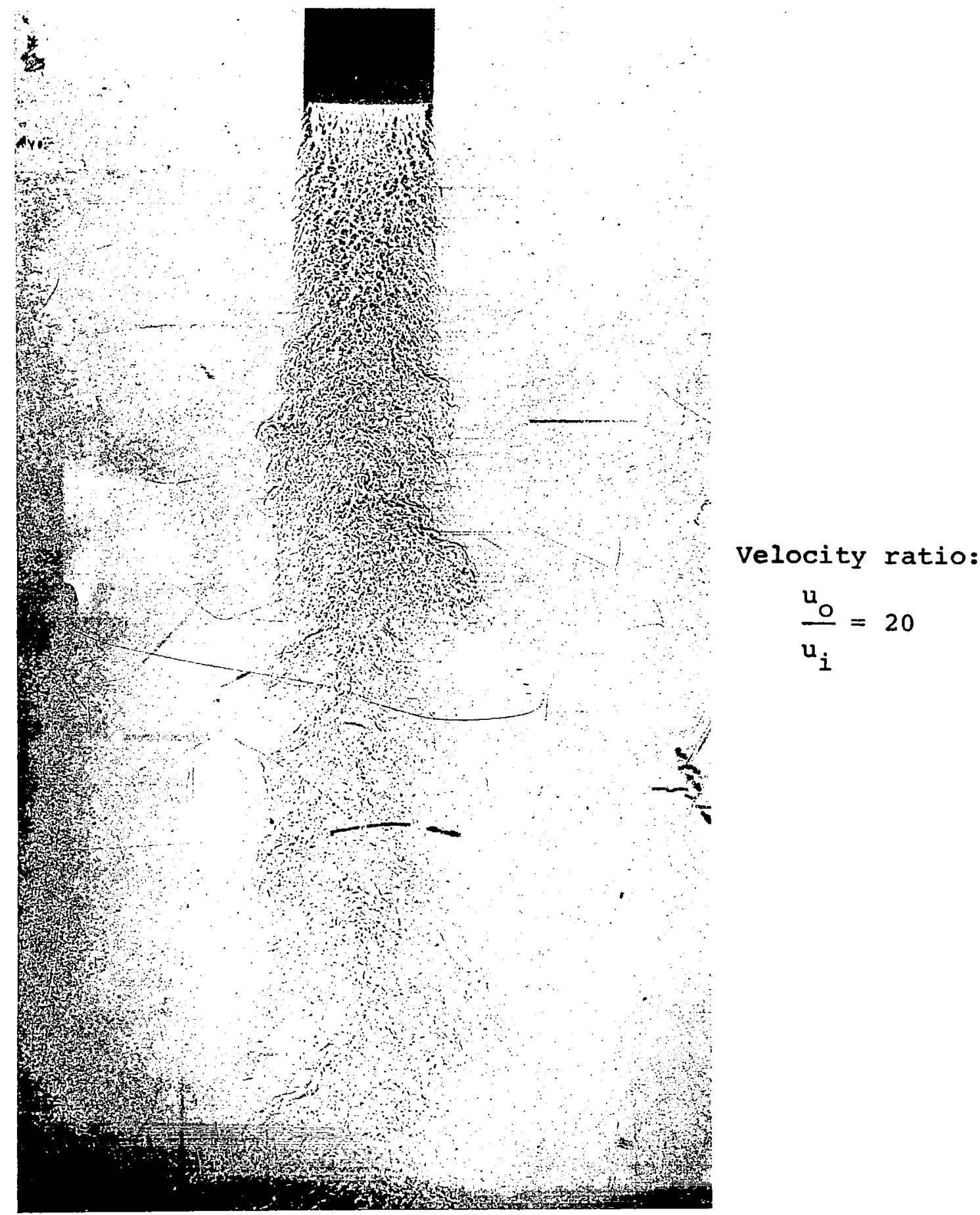

Freon jet issuing into moving air stream

Plate 4 
reports a value of $c=(.2-.3)$ for a submerged jet. It is shown here that for heterogeneous coaxial jets with density ratio of 4 and the outer stream moving faster than the inner stream, the value of $C$ is an order of magnitude smaller than this value. However, changing density ratio should have little effect on the spreading rate and therefore the moving outer stream retards the spreading as suggested by Abramovich. Thus the spread of a jet in a faster moving outer stream is slower than the spread of a coaxial jet with slower moving outer stream or submerged jet. Plate 5 shows the difference in spread of a 2:1 velocity ratio coaxial jet and the submerged jet of the same inner stream velocity. It can be clearly seen that the submerged jet spreads faster than the coaxial jet.

When the inner stream is faster than the outer stream, the direction of material transfer is the same as the direction of momentum transfer - from the faster to the slower moving jet. In the present case, momenturn is transferred from the outer faster stream to the slower heavier Freon jet. Thus, the large decrease in Freon concentration (figure 11 ) is not a result of fast diffusion of Freon into air, but is a result of bulk movement of the outer air stream toward the center line. The merging of the faster air stream with the inner freon jet results in the dilution of the inner stream. This dilution takes place to a great extent in the initial region. Plates 5 to 11 show the initial region of the heterogeneous jet for 

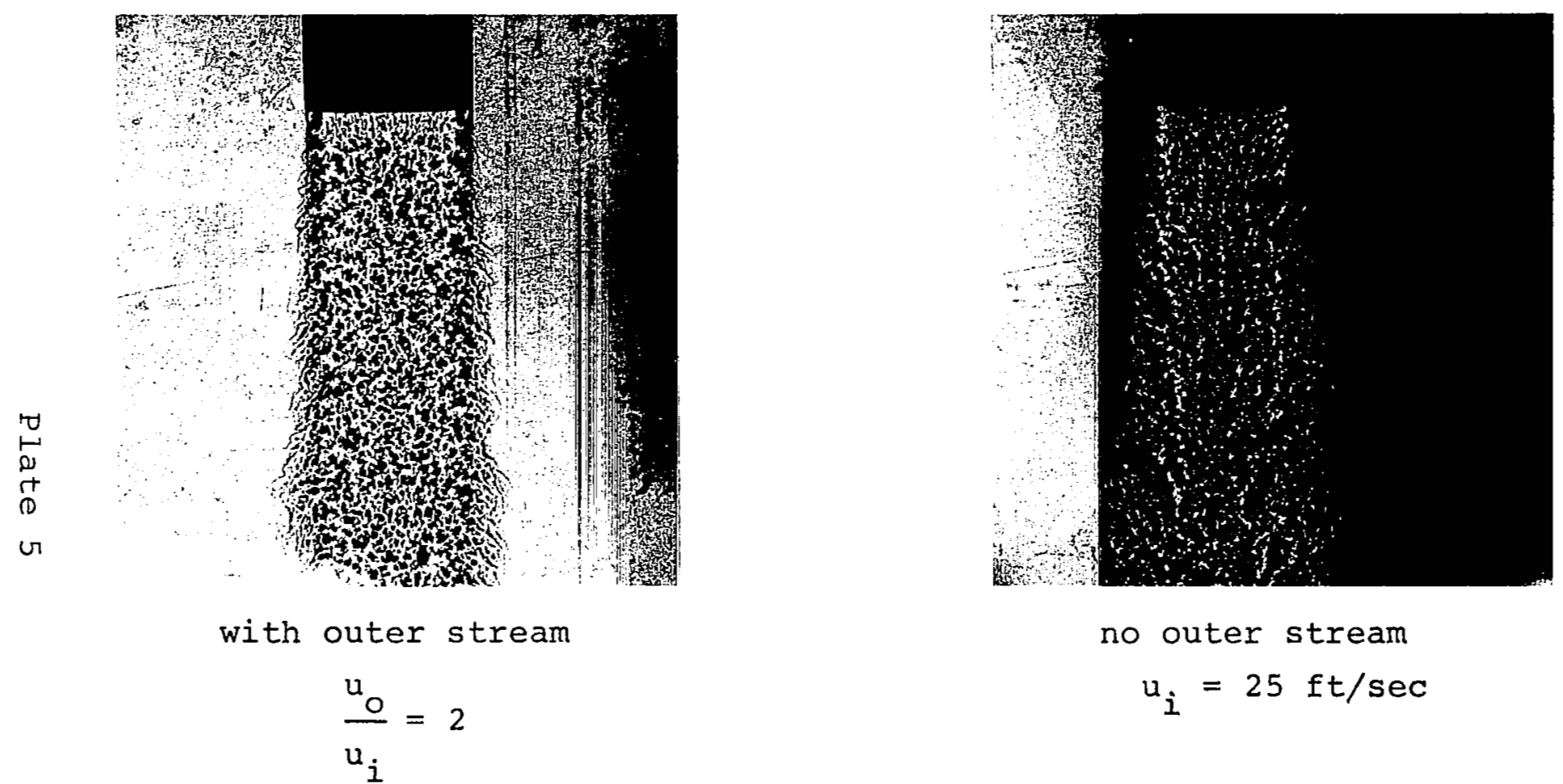

initial region of Freon jet into moving air stream 


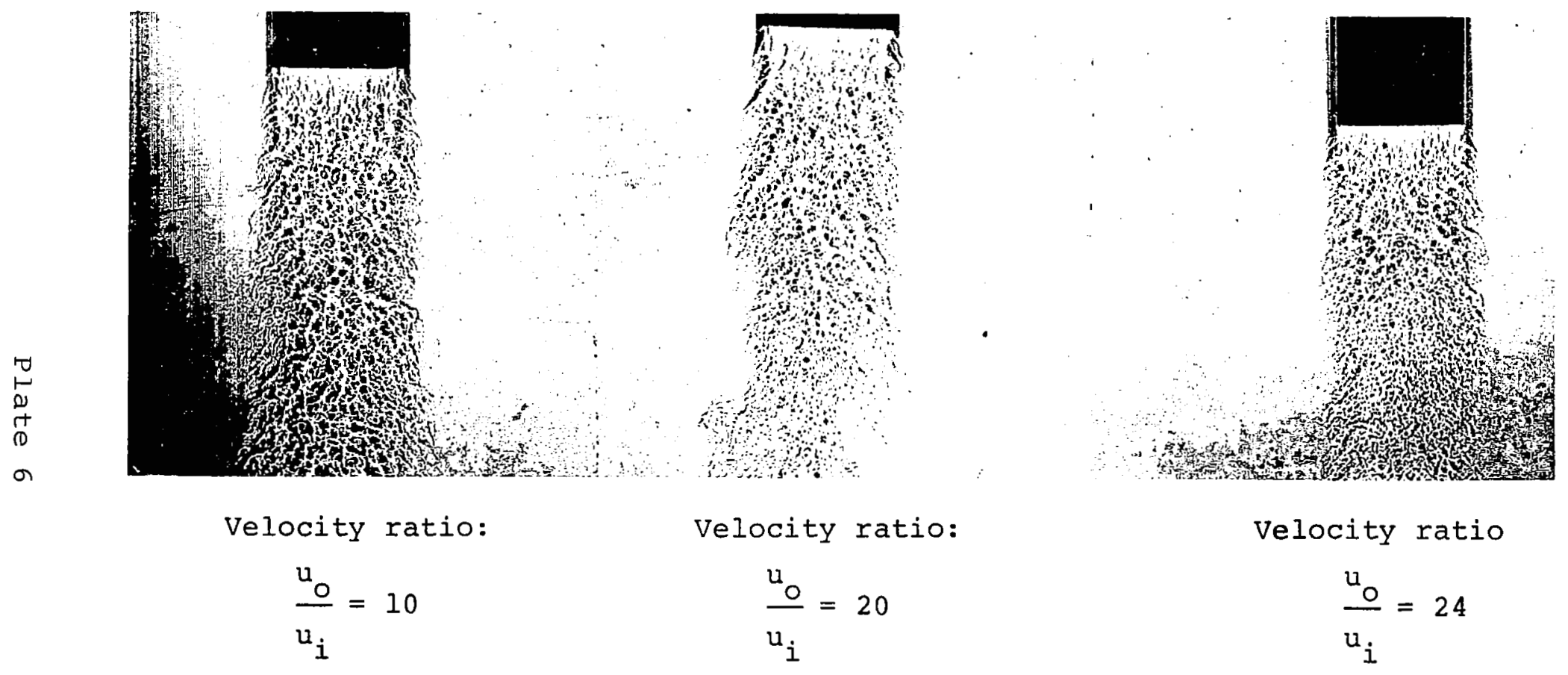

initial region of Freon jet into moving air stream 

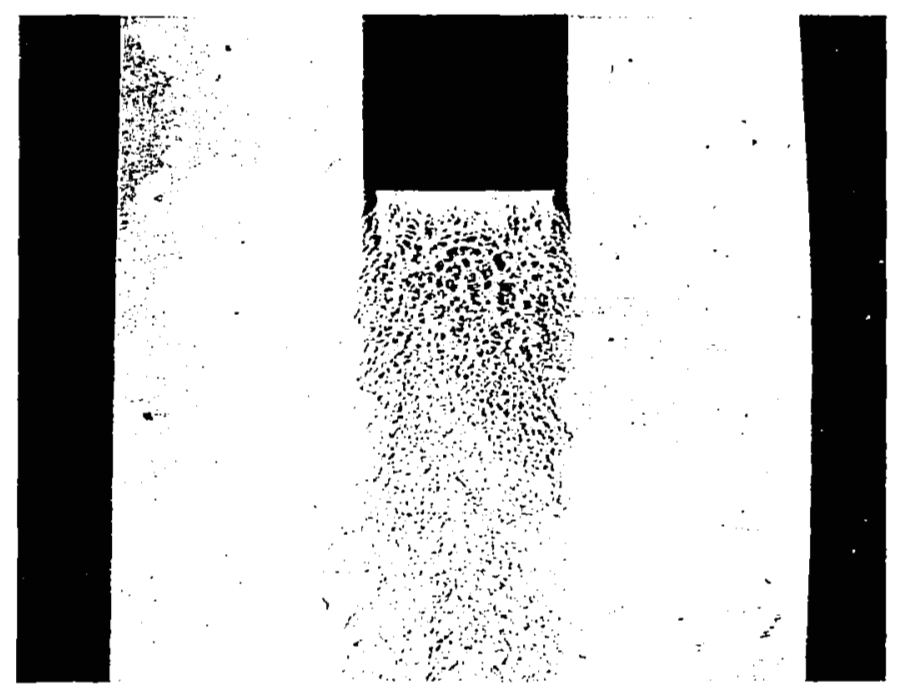

Velocity ratio
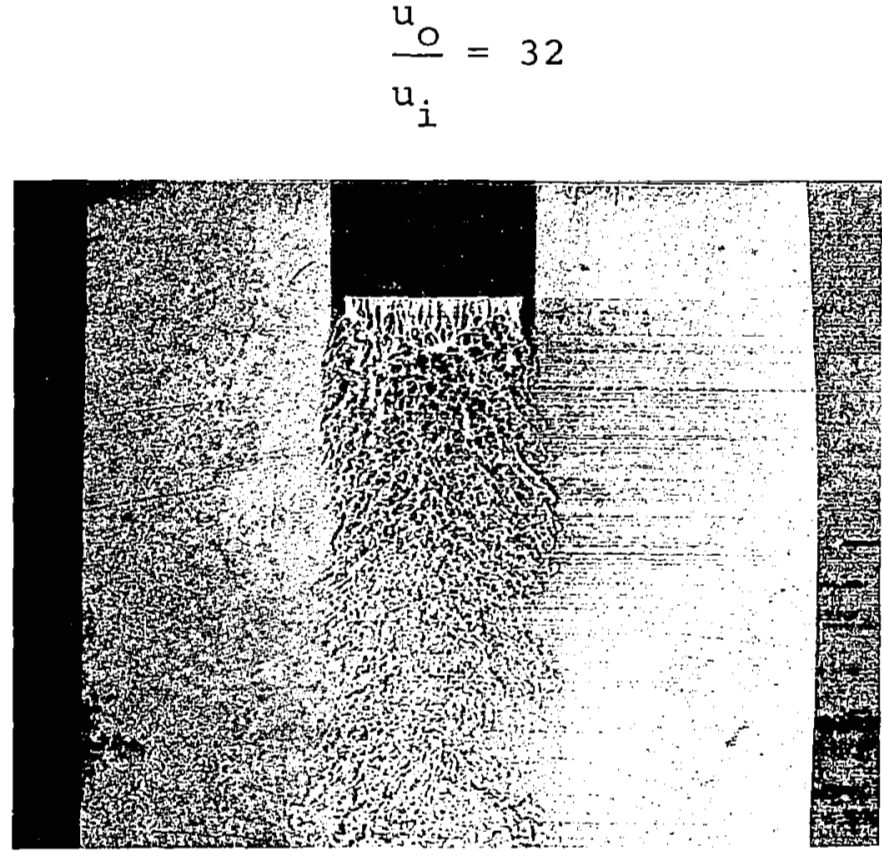

Circulation in the initial region of

Freon Jet issuing into moving air stream

Plate 7 


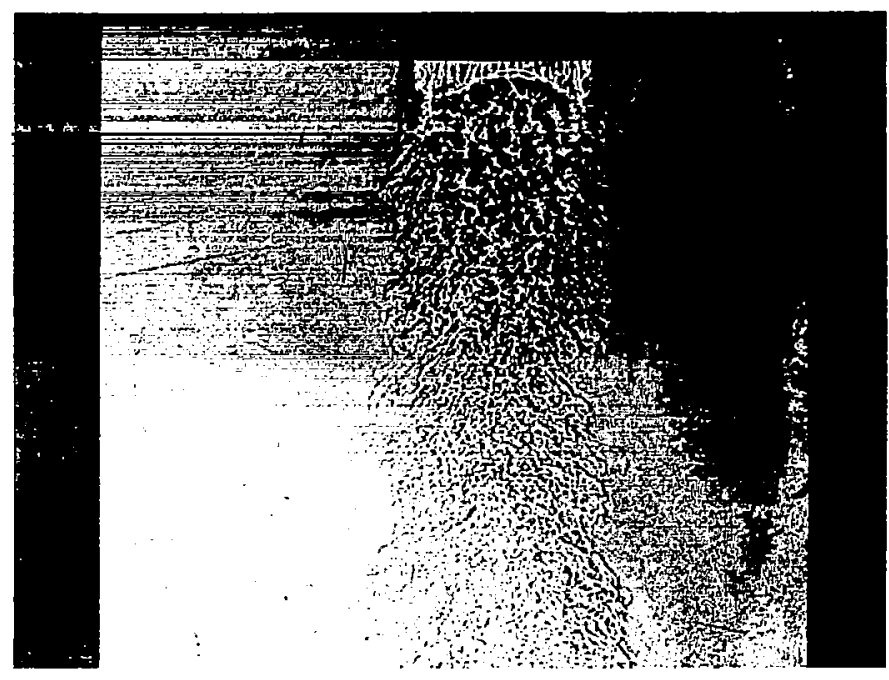

Velocity ratio:

$\frac{u_{0}}{u_{i}}=35$

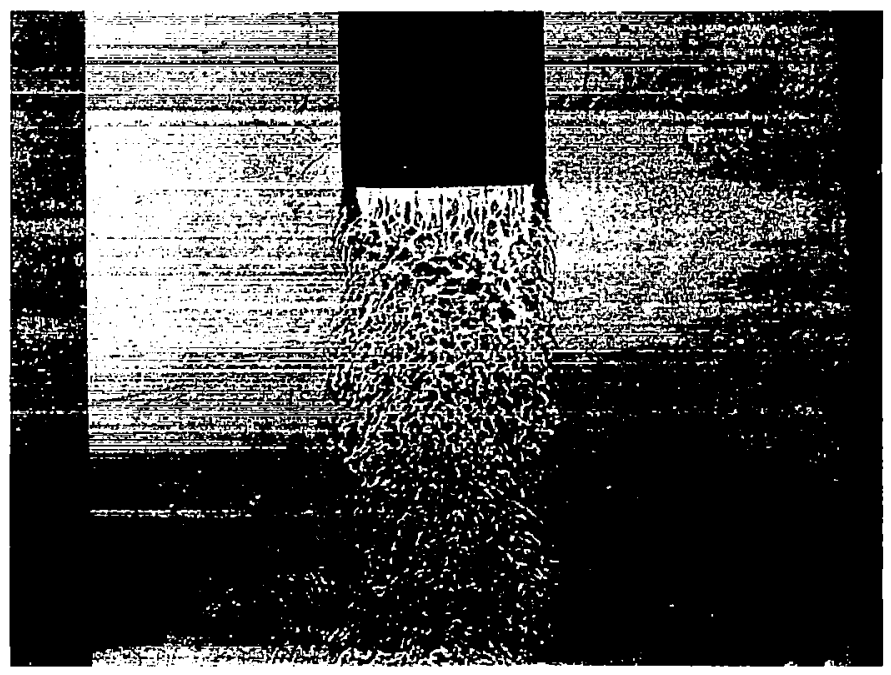

Circulation in the initial region of Freon Jet issuing into moving air stream Plate 8 


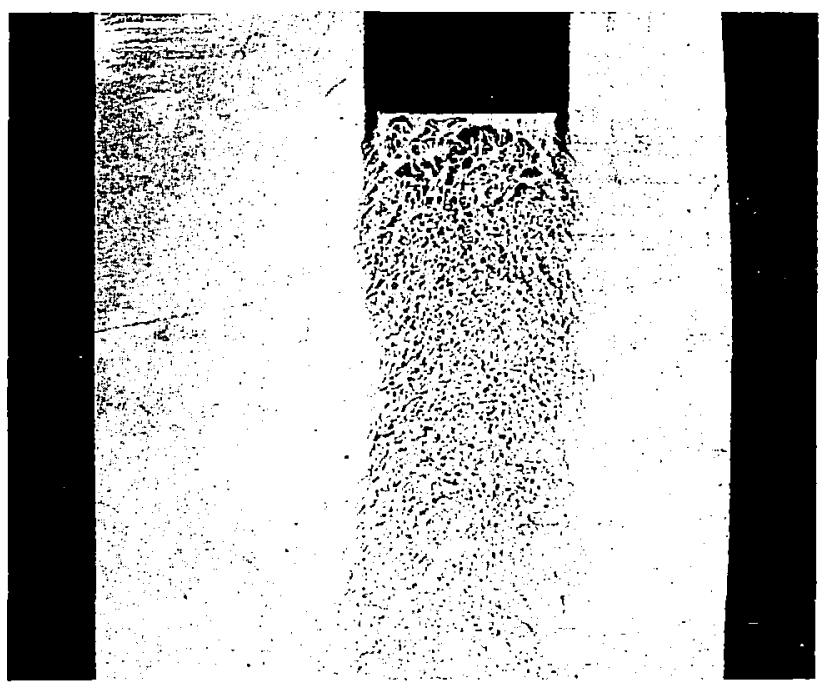

Velocity ratio:

$\underline{u_{0}}=37.5$

$u_{i}$

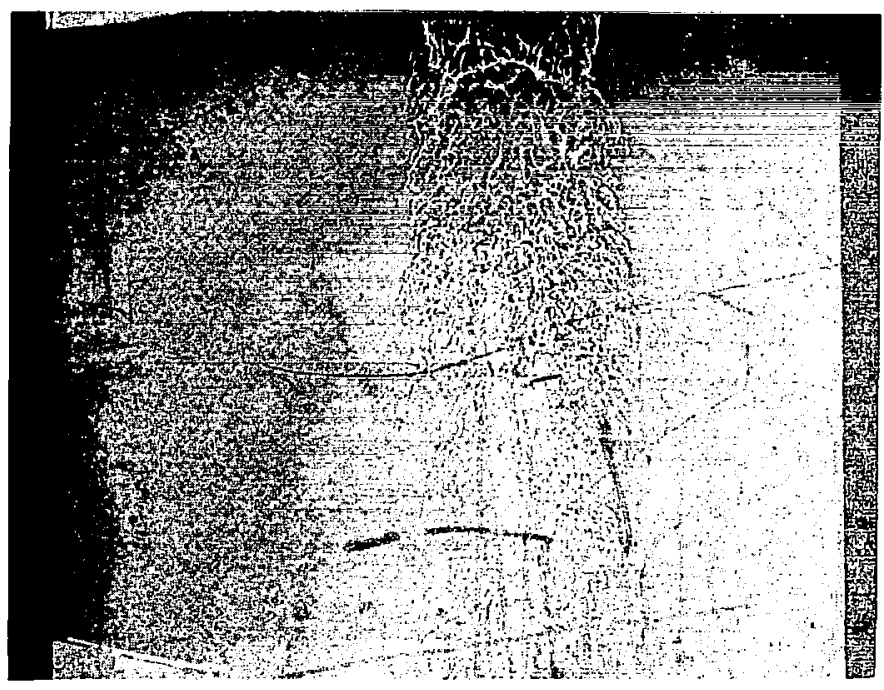

Circulation in the initial region of

Freon Jet issuing into moving air stream

Plate 9 


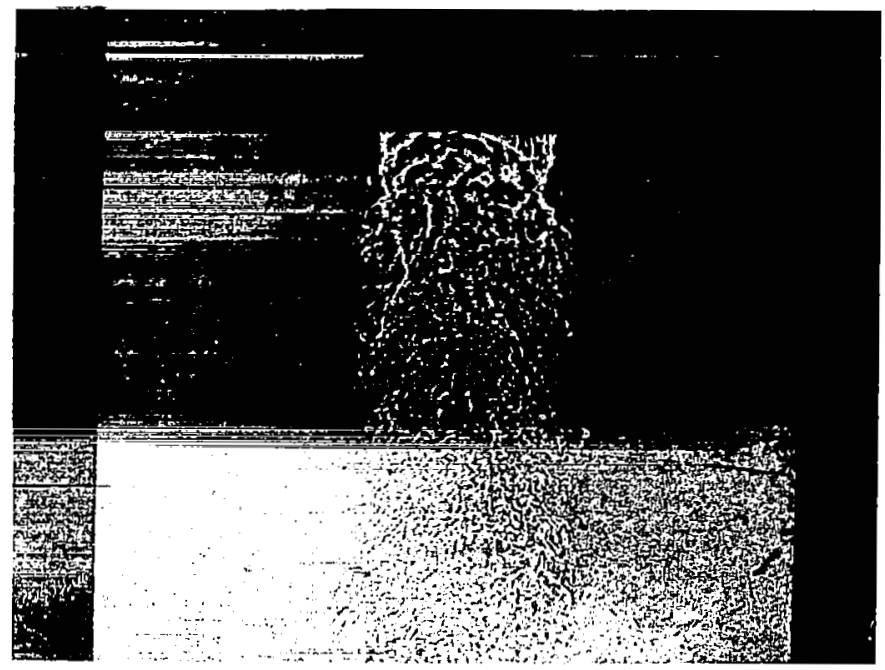

$$
\frac{\mathrm{U}_{\mathrm{O}}}{\mathrm{U}_{\mathrm{i}}}=40
$$
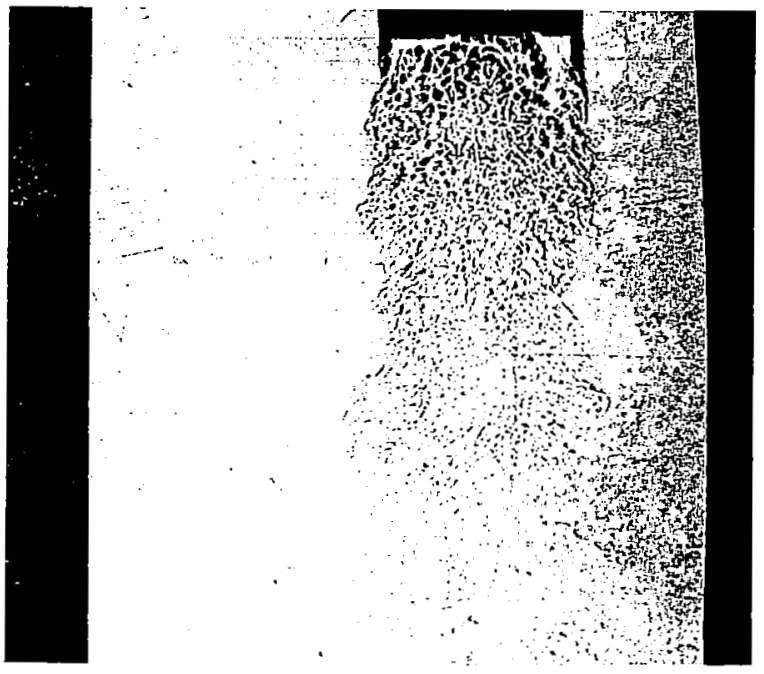

Circulation in the initial region of

Freon Jet issuing into moving air stream

Plate 10 

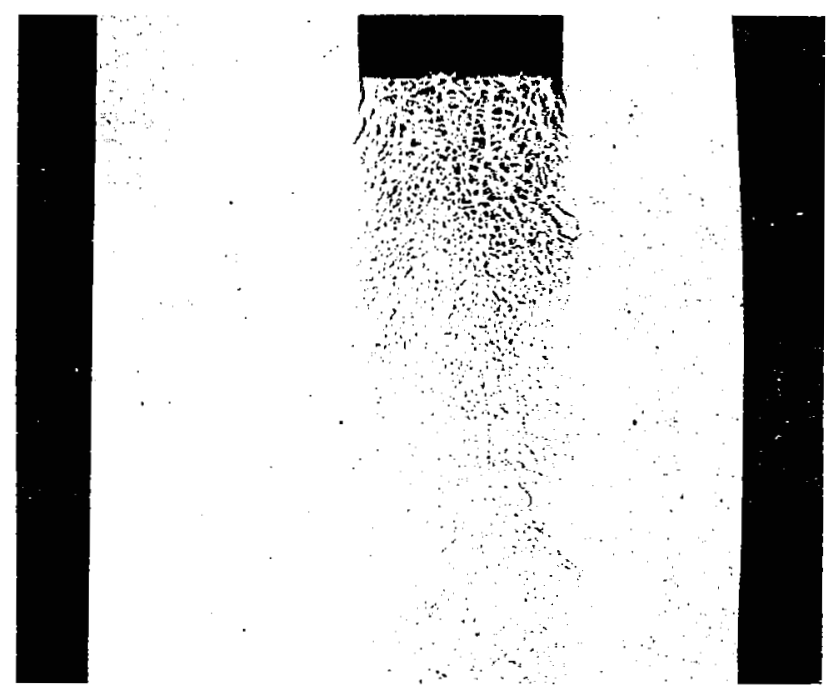

Velocity ratio:
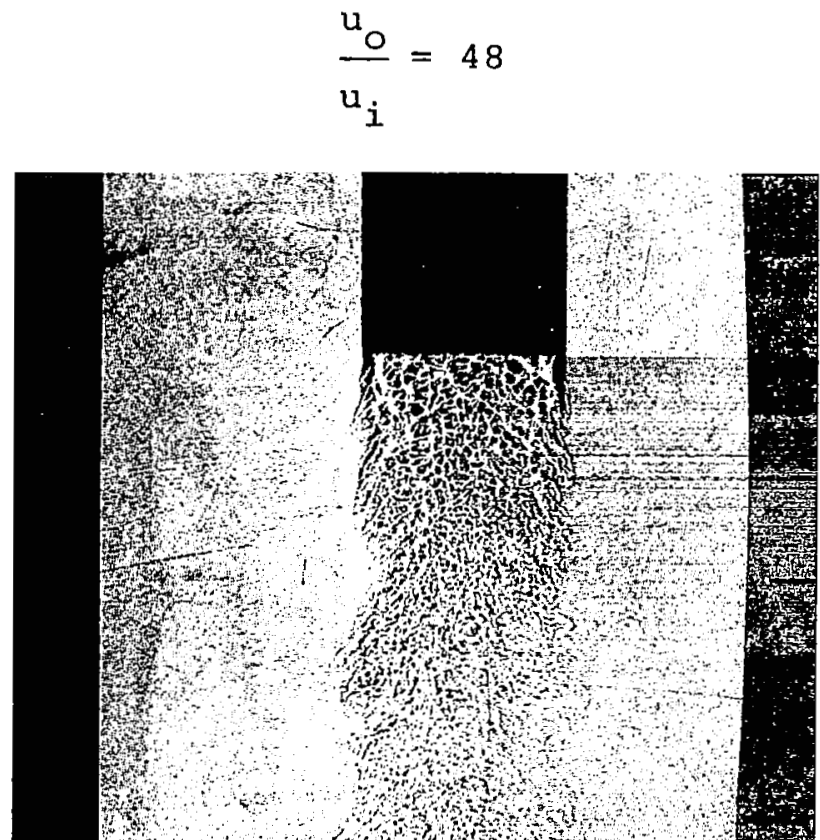

Circulation in the initial region of

Freon Jet issuing into moving air stream Plate 11 
increasing velocity ratio. With the cylindrical probe it was found that back flow started at $U_{0} / U_{i}=13$ for the homogeneous jet and $U_{0} / U_{i}=26$ for the heterogeneuos jet. Plates 7 to 11 show the initial region with back flow. The back flow region moves upstream with the increase in velocity ratio, and for $U_{0} / U_{i}=40$ (plate 10 ) it is evident that the back flow region penetrates into the pipe.

Plate 12 demonstrates the back flow region in the homogeneous jet. Small quantities of Freon were injected through a hypodermic tube at the center line of the inner air jet. The pattern of the Freon tracer is visible on the shadowgraph. The upper picture in the plate shows the path of the tracer in the inner jet with no outer stream. The pictures on the bottom of the plate show the path of the Freon with the outer stream. It is clearly seen that the Freon tracer which was previously undisturbed, forms a turbulent bubble.

The tracer impinges on a mountain of high pressure and is spread in all directions. This indicates that for these velocity ratios a vortex back flow region exists near the nozzle exit. The issuing stream separates from the center line and flows around the vortex. However, the Freon tracer jet with its slightly larger than inner stream velocity penetrates the backflow region somewhat before being turned around and dispersed by the backflow. See 

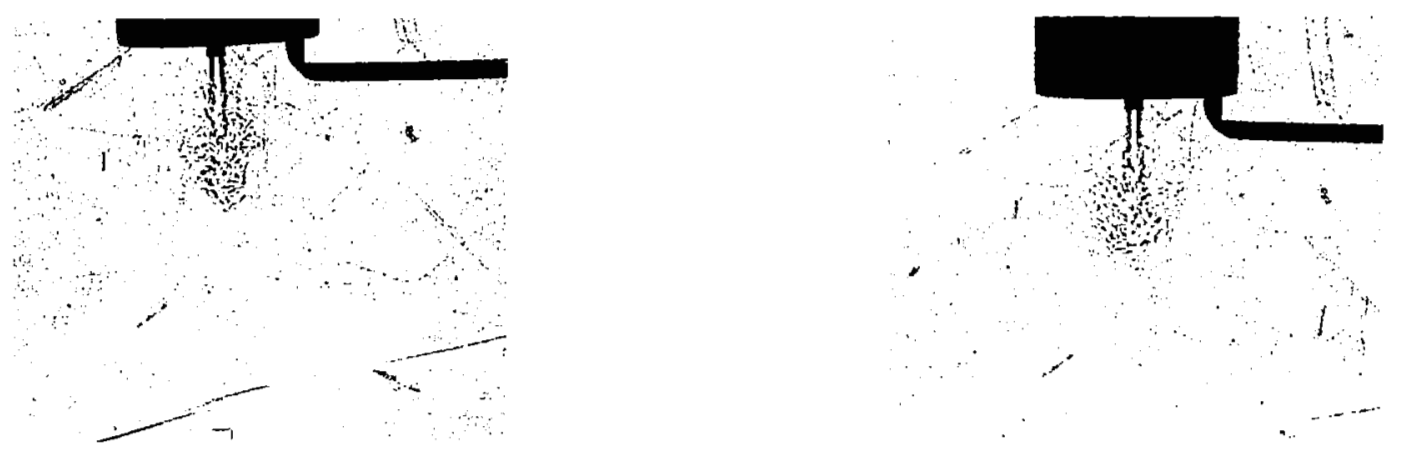

Velocity Ratio

$$
\frac{\text { Uo }}{\text { Ui }}=25
$$

Tracer Injection In Back Flow Region

Plate 12 

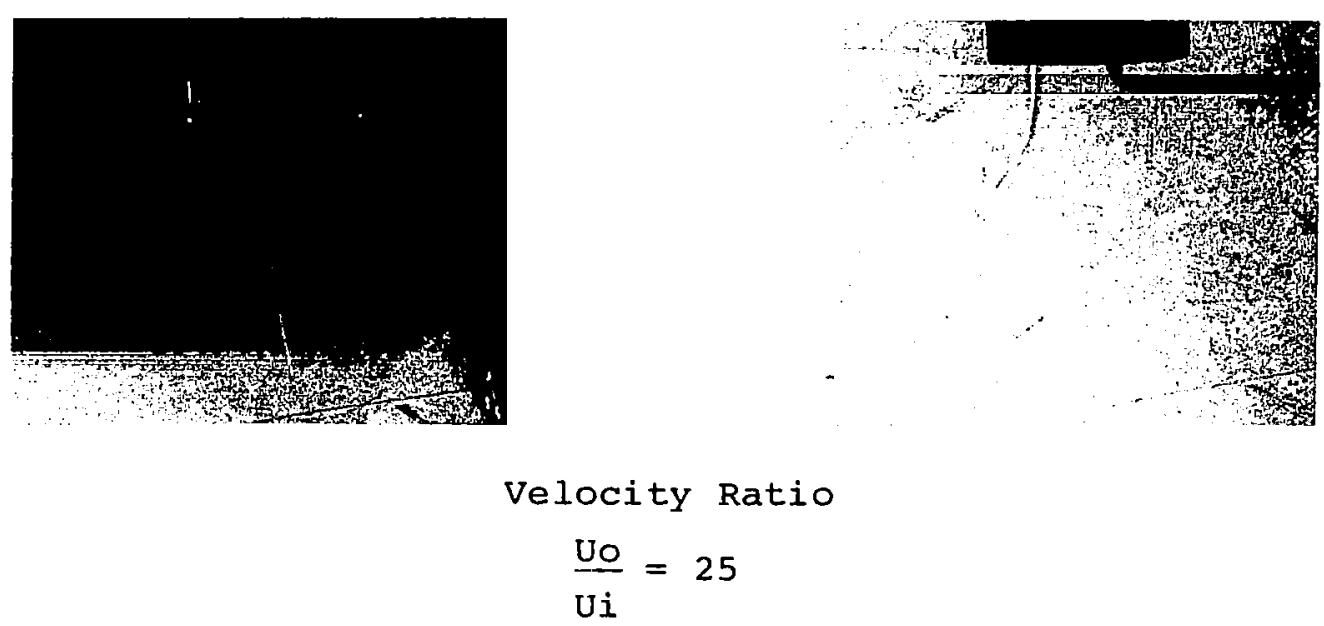

Tracer Injection In Back Flow Region

Plate 13 
figure 12. Plate 13 shows the path of the Freon tracer injected at a radial location of $r / r_{0}=.5$ without outer stream on the left and with outer stream on the right. The path of the tracer follows the assumed stream lines and turns around the established vortex.

From the shadowgraphs, the existence of back flow region was considered as established and measurements were carried out in the initial region for the velocity ratios for which back flow exists. Before making actual measurements of velocity, the location of the downstream stagnation point was found with the aid of the cylindrical probe. The velocity ratio for which a first stagnation point was found was $U_{O} / U_{i} \cong 13$. The stagnation point was at the downstream distance of approximately $x / r_{0}=2.8$. The local turbulence intensity was quite large.

Figure 13 shows the axial velocity on the center line of the jet for high velocity ratios $\mathrm{U}_{0} / \mathrm{U}_{i}=16,32$. Figure 14 shows the distribution of the corresponding axial turbulence intensities. Figure 14 exhibits two peaks in the turbulence intensity profile which indicates the existence of unstable regions with a relatively stable flow region "locked" between them. The downstream peaks in the turbulence intensity correspond to the points where the axial velocity is roughly zero - namely the stagnation point. From figure 13 it is evident that for velocity ratios $\mathrm{U}_{\mathrm{O}} / \mathrm{U}_{i}=\infty$ and 32 , the upstream stagnation point is in the pipe, whereas from figure 14, the maximum turbulence 


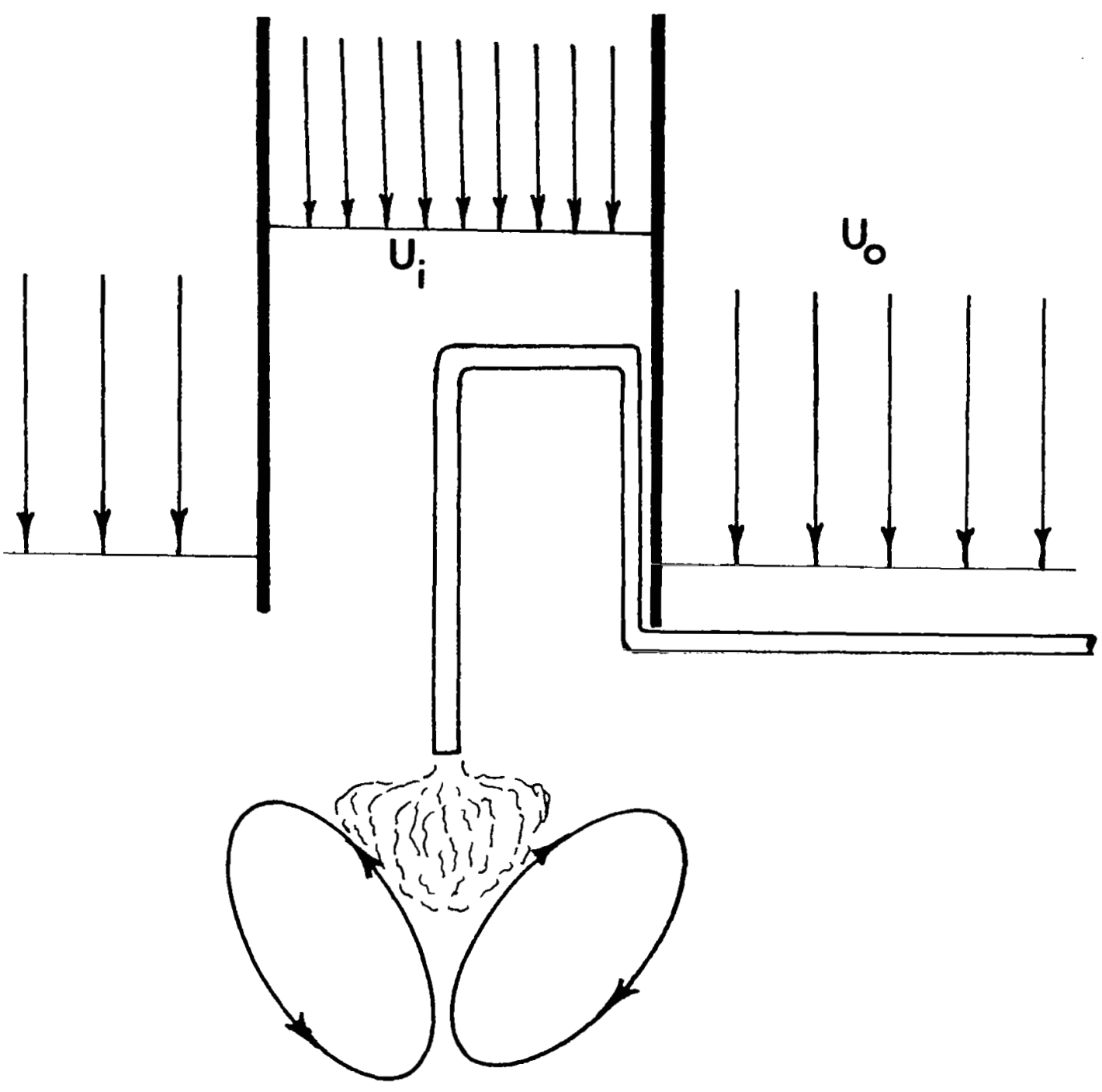

Figure 12. - Distortion of recirculation region by tracer jet. 


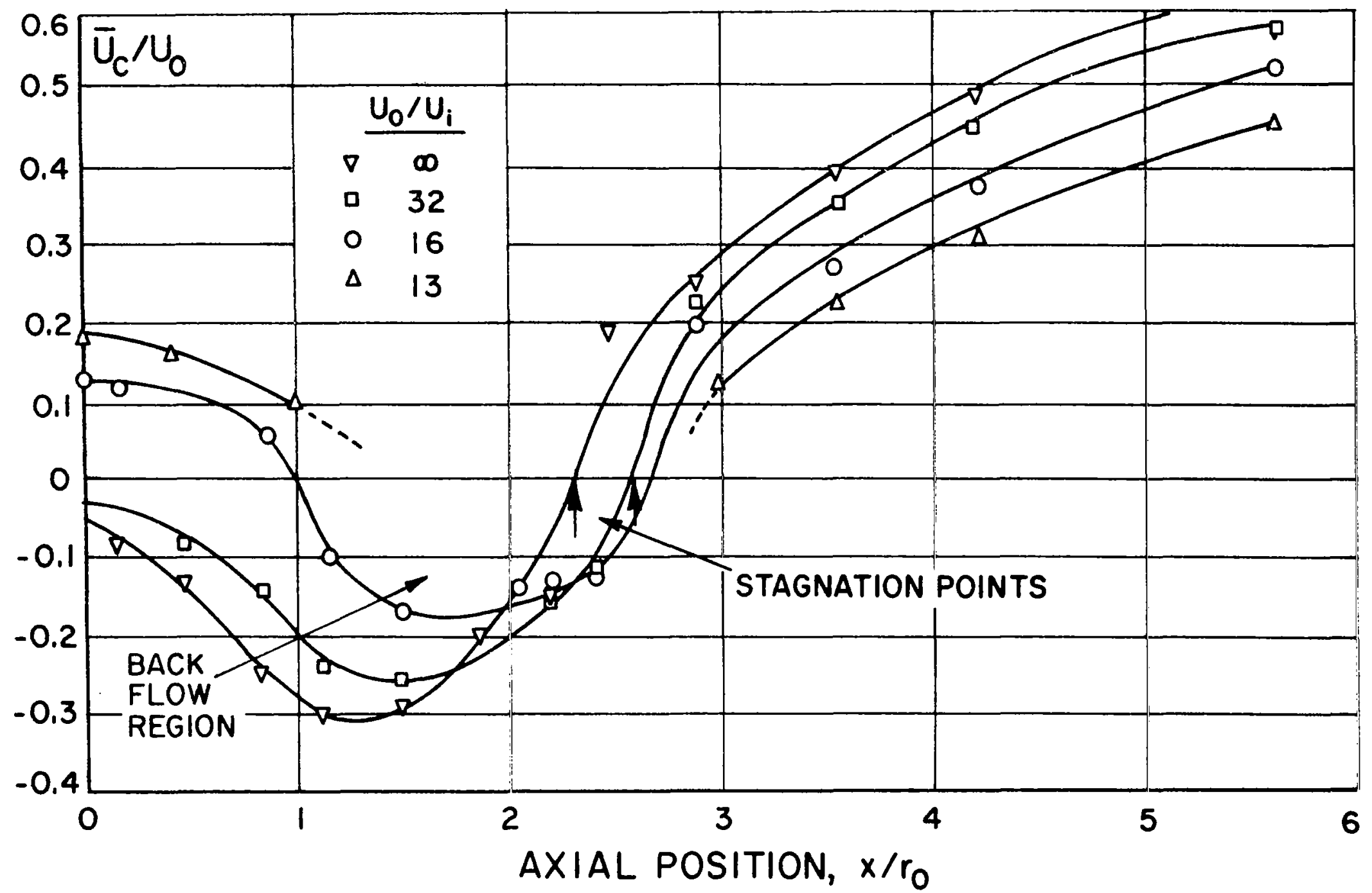

Figure 13. - Distribution of axial velocity on the centerline of the jet. 


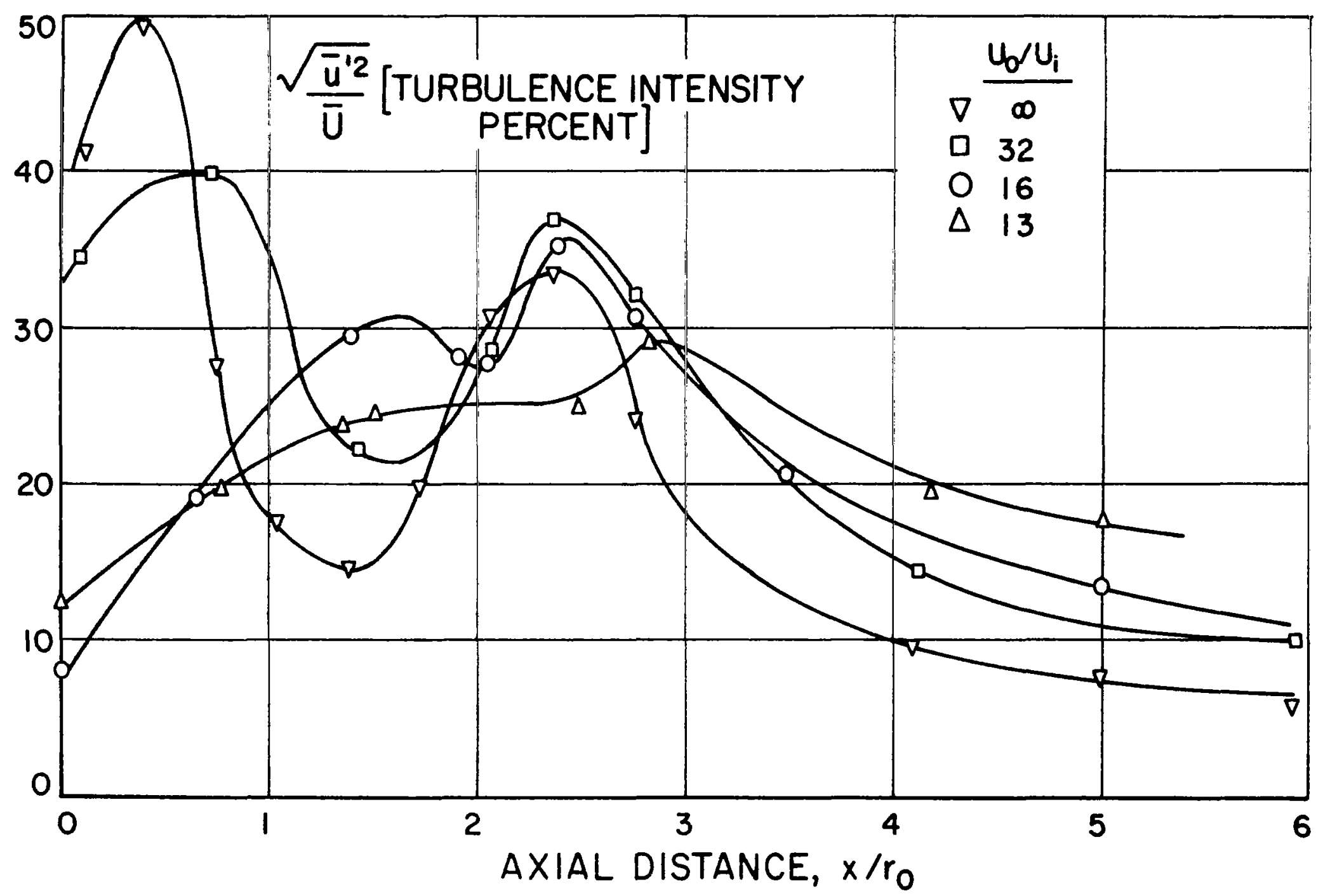

Figure 14. - Distribution of axial turbulence on centerline. 
intensity is approximately at $x / r_{0}=.5$. This discrepancy is in part a result of high turbulence generated near the pipe exit. No turbulence measurements were taken in the inside of the pipe. It appears as if the downstream stagnation point is more stable than the upstream stagnation point and hence, the upstream portion of the vortex is probably very unstable. Figure 14 also shows that a low velocity inner jet is accelerated faster than a higher velocity jet.

Assuming that the stream lines are parallel to the center line at the axial station of the vortex center, the hot wire readings at this location were taken to represent the local axial velocity. Figures 15 and 16 show the axial velocities and turbulence intensities as a function of radial distance. As the velocity ratio increases the vortex center moves away from the center line increasing the diameter of the vortex.

Figure 17 shows the distribution of center line pressure as a function of velocity ratio. Data for five velocity ratios is given, $U_{0} / U_{i}=\infty, 28.5,18,10.8,6$.

Back flow existed for the first three velocity ratios. The pressure plot for $U_{0} / U_{i}=\infty, 28.5,18$ exhibit a similar form. The pressure increases upstream of the initial face reaching a peak, then decreases rapidly to a minimum and increases again to a second maximum. Similar pressure results were obtained by Calvert (14) who measured pressure distribution in the initial region of a wake behind 


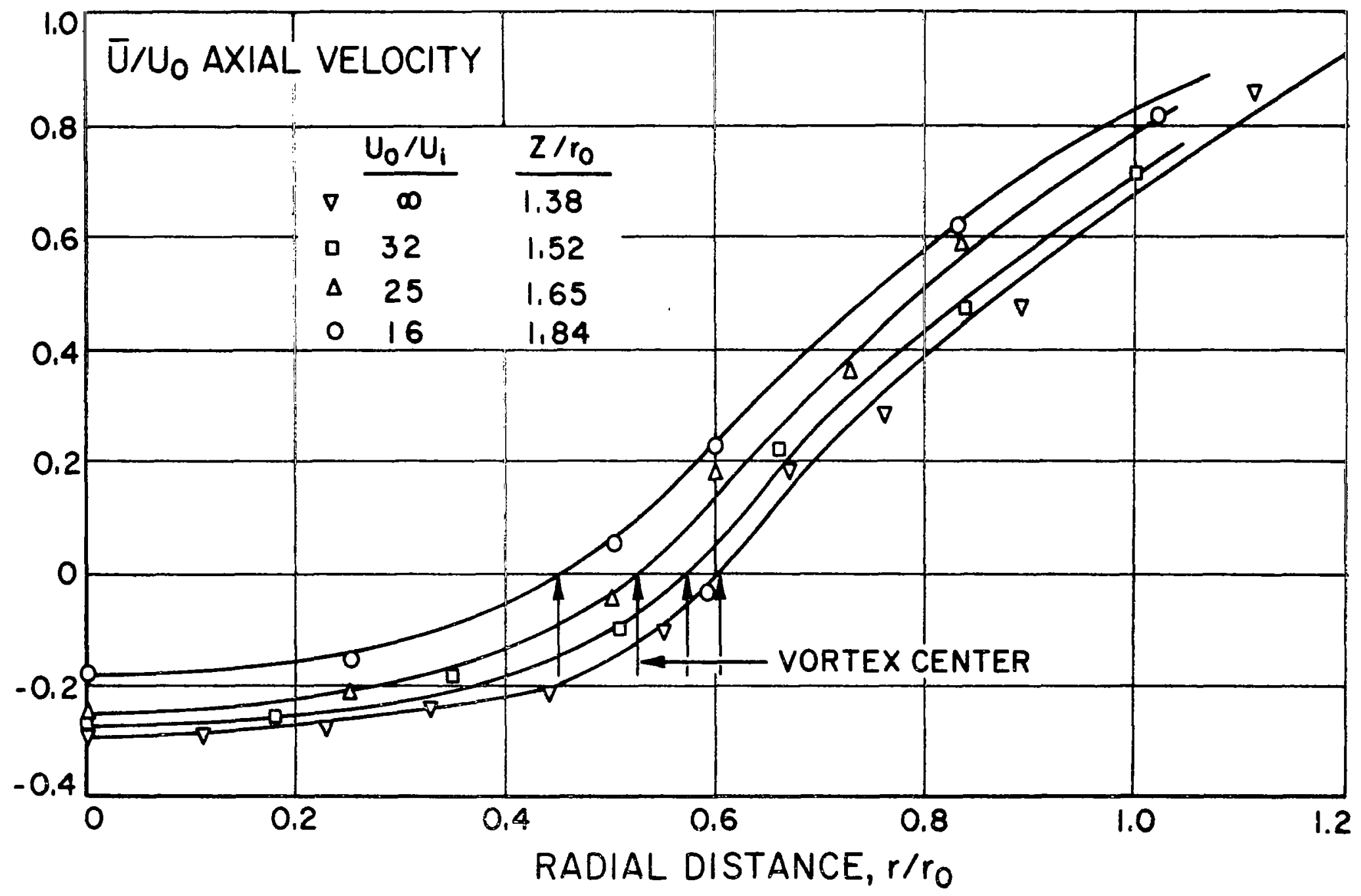

Figure 15. - Radial distribution of axial velocity at the axial position of the vortex center. 


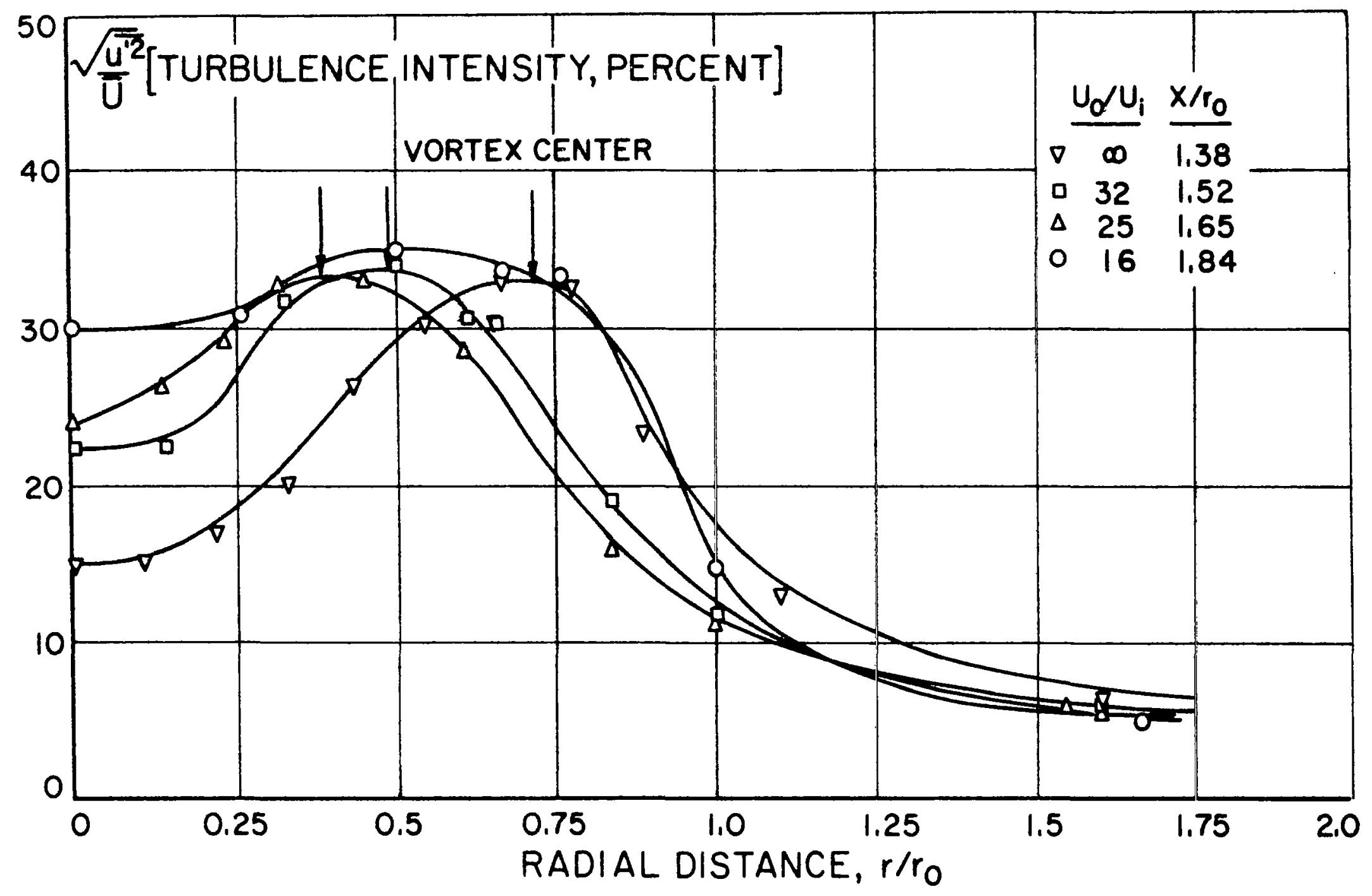

Figure 16. - Radial distribution of axial turbulence at the axial position of the vortex center. 


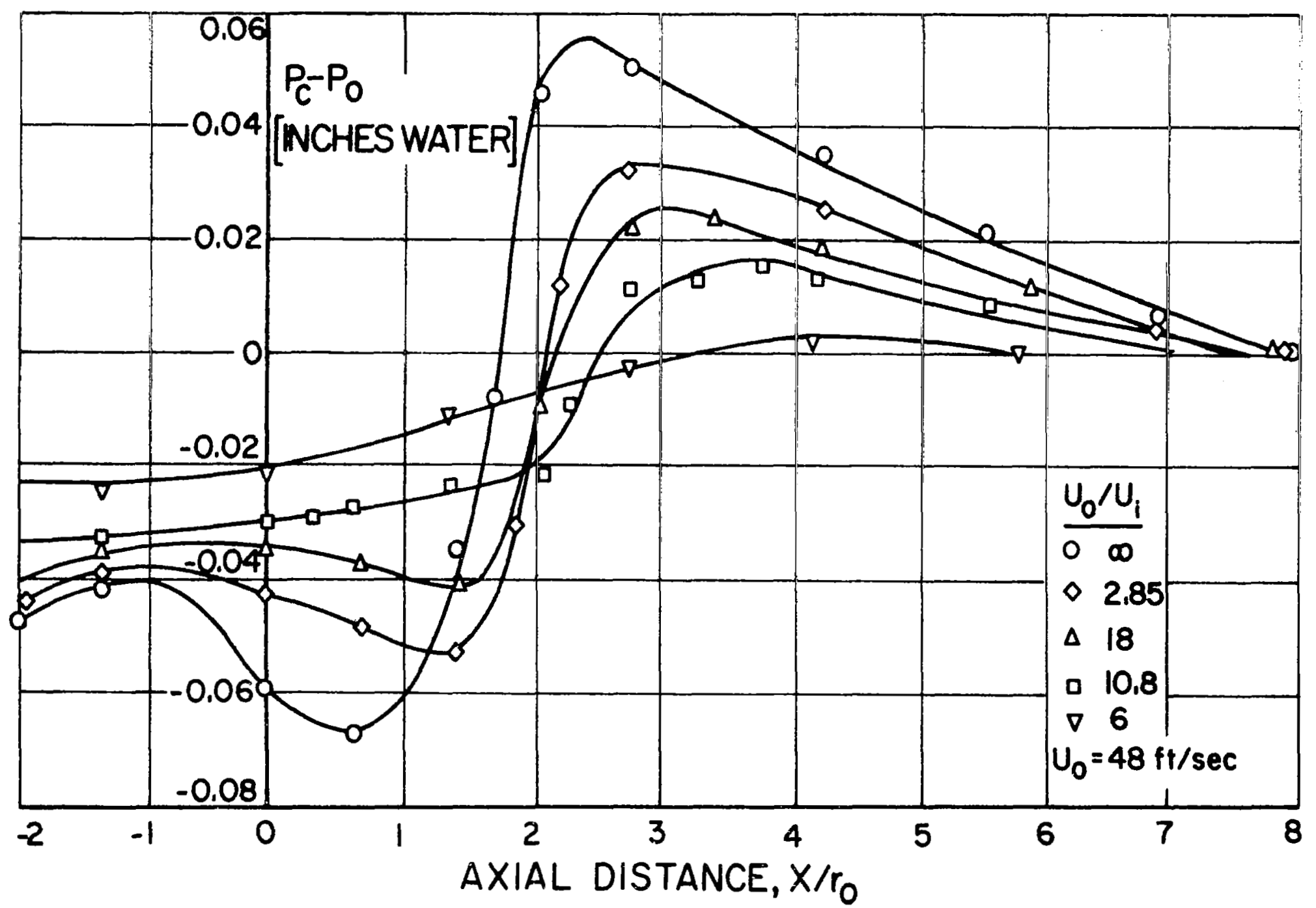

Figure 17. - Centerline pressure versus velocity ratio, homogeneous jet. 
cones of different angles. The pressure plots for $\mathrm{U}_{\mathrm{O}} / \mathrm{U}_{i}=10.8$ and 6 display only a maximum with no minimum. For the cases where circulation was present, the downstream maximum is the location of the stagnation point. The maximum value of the pressure is higher for higher velocity ratios. For $\mathrm{U}_{\mathrm{O}} / \mathrm{U}_{\mathrm{i}}=\infty$ the maximum stagnation pressure is .056 inches of water corresponding to $.14\left(1 / 2 \rho_{0}{ }^{2}\right)$ or $14 \%$ of the total dynamic head of the outer stream. Figure 18 shows the corresponding curves of velocity, turbulence intensity and pressure for $\mathrm{U}_{\mathrm{O}} / \mathrm{U}_{i}=\infty$. The consistency is satisfactory. The pressure for $\mathrm{U}_{\mathrm{O}} / \mathrm{U}_{\mathrm{i}}=10.8$ and 6 increases monotonically, reaches a maximum and decreases, indicating that the center line velocity decreases monotonically in the downstream direction and reaches a minimum. The location of the minimum velocity corresponds to the location of the maximum in the pressure plot. For velocity ratio smaller than 3, no pressure difference was observed. Figure 19 shows the pressure plot for the heterogeneous jet. Data for three velocity ratios, $U_{O} / U_{i}=50,36,26$ are given. For the two velocity ratios of 50 and 36 back flow is established while for $U_{0} / U_{i}=26$ back flow is incipient. The pressure plots in this case are similar to the homogeneous case.

Correlation of Data

The measured velocity profiles could be correlated and made to fall on one line with suitable scale parameters. 


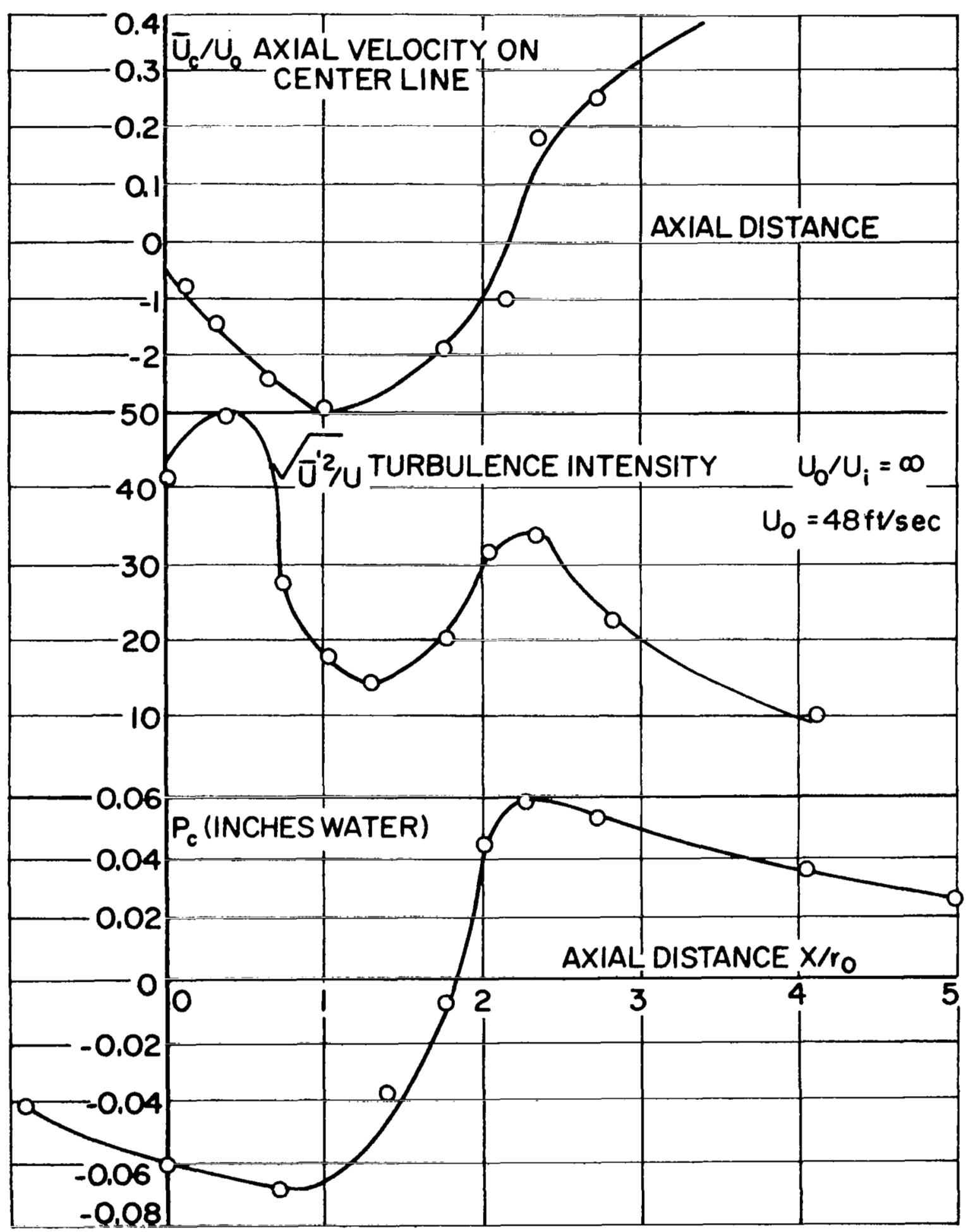

Figure 18. - Pressure, velocity, and turbulence intensity on centerline of the jet. 


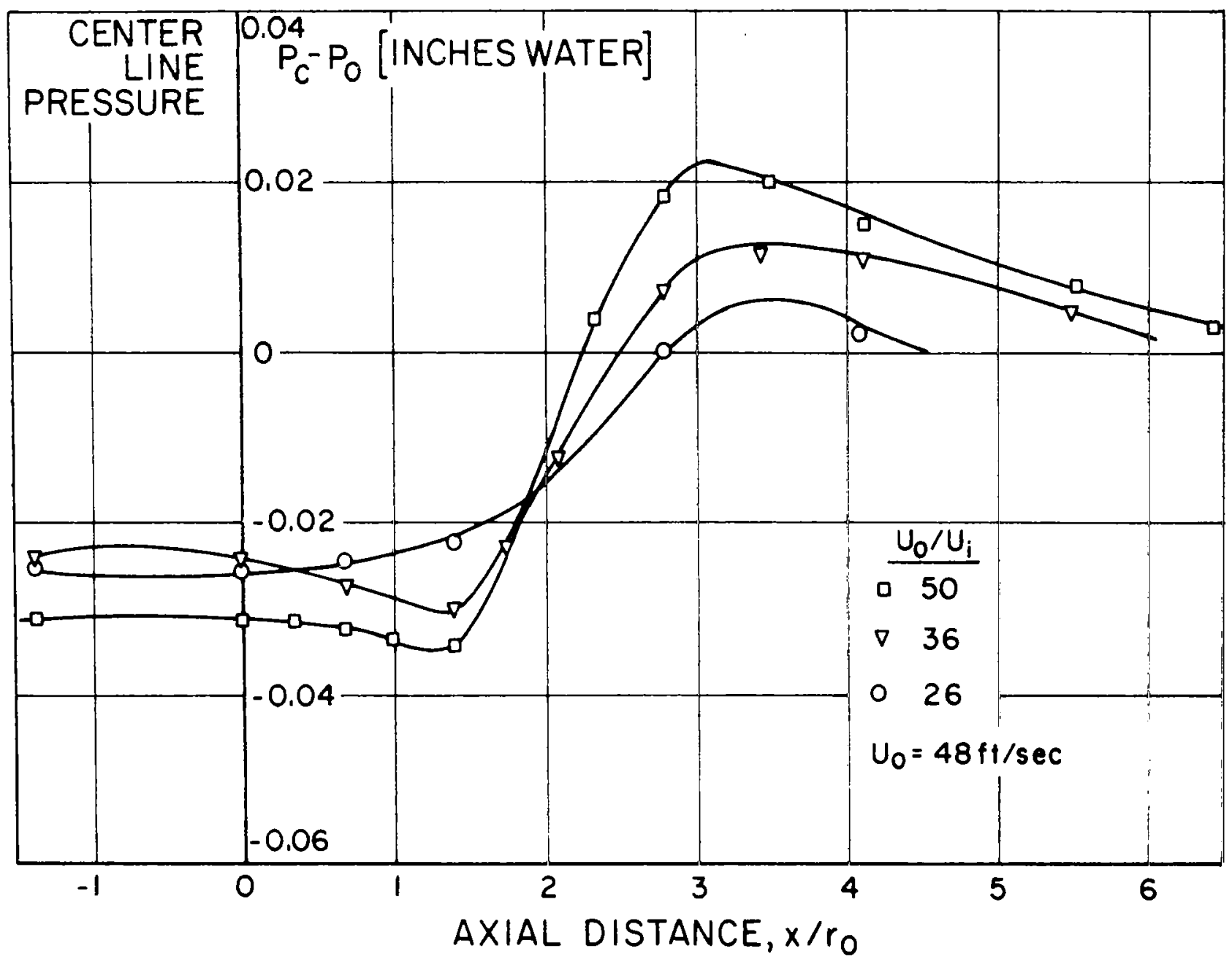

Figure 19. - Centerline pressure as a function of velocity ratio, heterogeneous jet, $p_{0} / p_{i}=4$. 
Figure 20 shows a plot of non-dimensional velocity $\mathrm{U} / \mathrm{U}_{\mathrm{min}}$ versus the nondimensional axial distance $x-x_{\min } / x_{\max }-x_{\min }$ where $x_{\max }$ is the location of the downstream stagnation point and $U_{\min }$, $x_{\min }$ are the maximum back flow velocity and its location in the vortex. The zero value of nondimensional distance corresponds to the location of the maximum back flow velocity. This figure shows that the established vortex is similar for different velocity ratios. The effect of velocity ratio manifests itself in establishing different maximum back flow velocities for different velocity ratios. However, the dimensionless velocity profile remains similar since the maximum backflow velocity is used as the velocity scale. Figure 21 shows a plot of the nondimensional axial velocity at the downstream location of the vortex center versus a nondimensional radial distance. The center line velocity which here is the maximum back flow velocity, was taken as the velocity scale, and the radial distance at which the axial velocity is zero was used as the length scale.

Figure 22 shows the comparison between pressure measurement by Calvert (14) and the present work. Calvert correlated his pressure results for different cones with a suitable definition of origin and streamwise length scale. The pressure scale was taken as the difference between the maximum and the minimum in each individual pressure plot, and the length scale was taken as the difference between the axial position at which the center line velocity was 


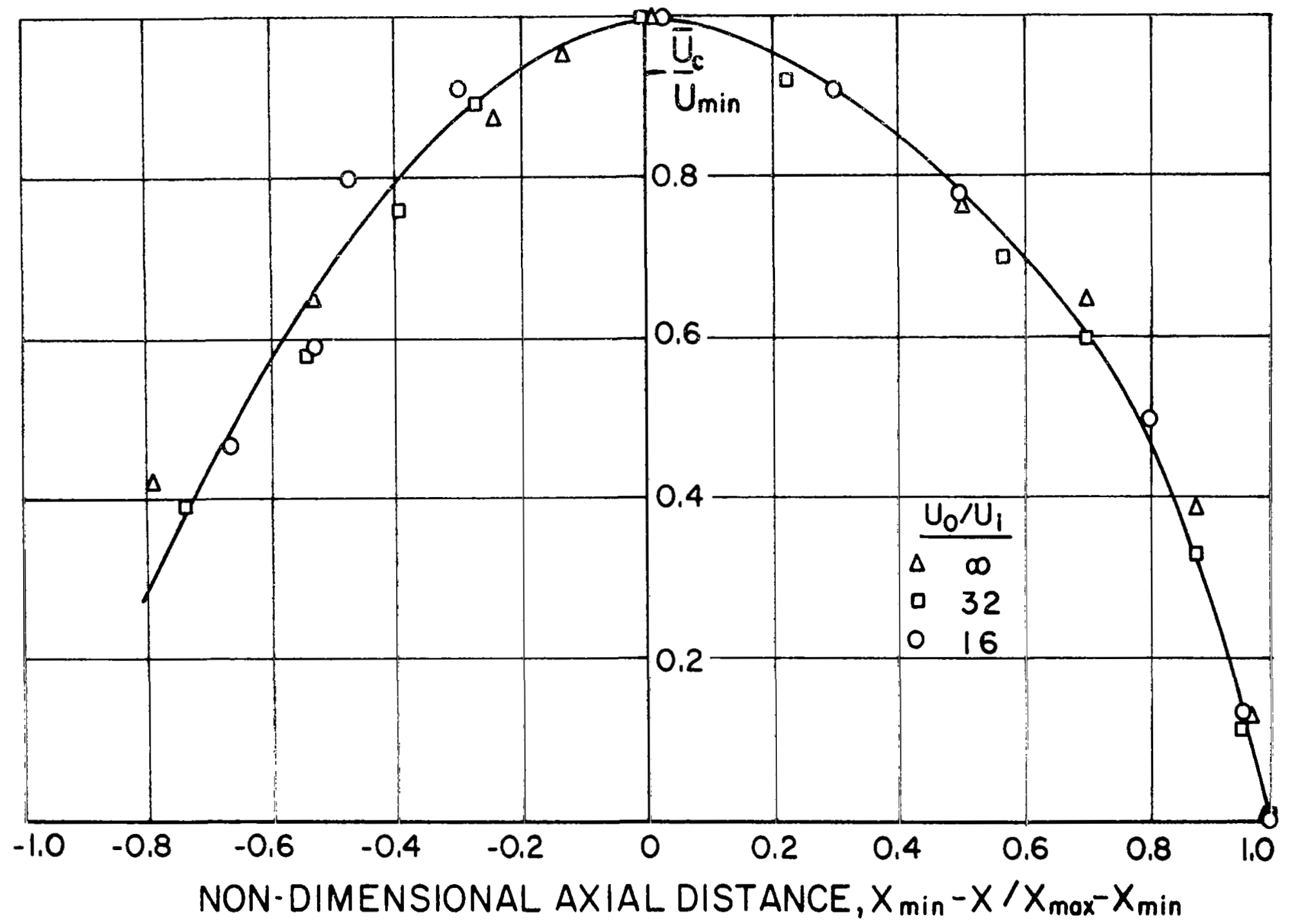

Figure 20. - Correlation of the centerline velocity in the backflow region for different velocity ratios. 


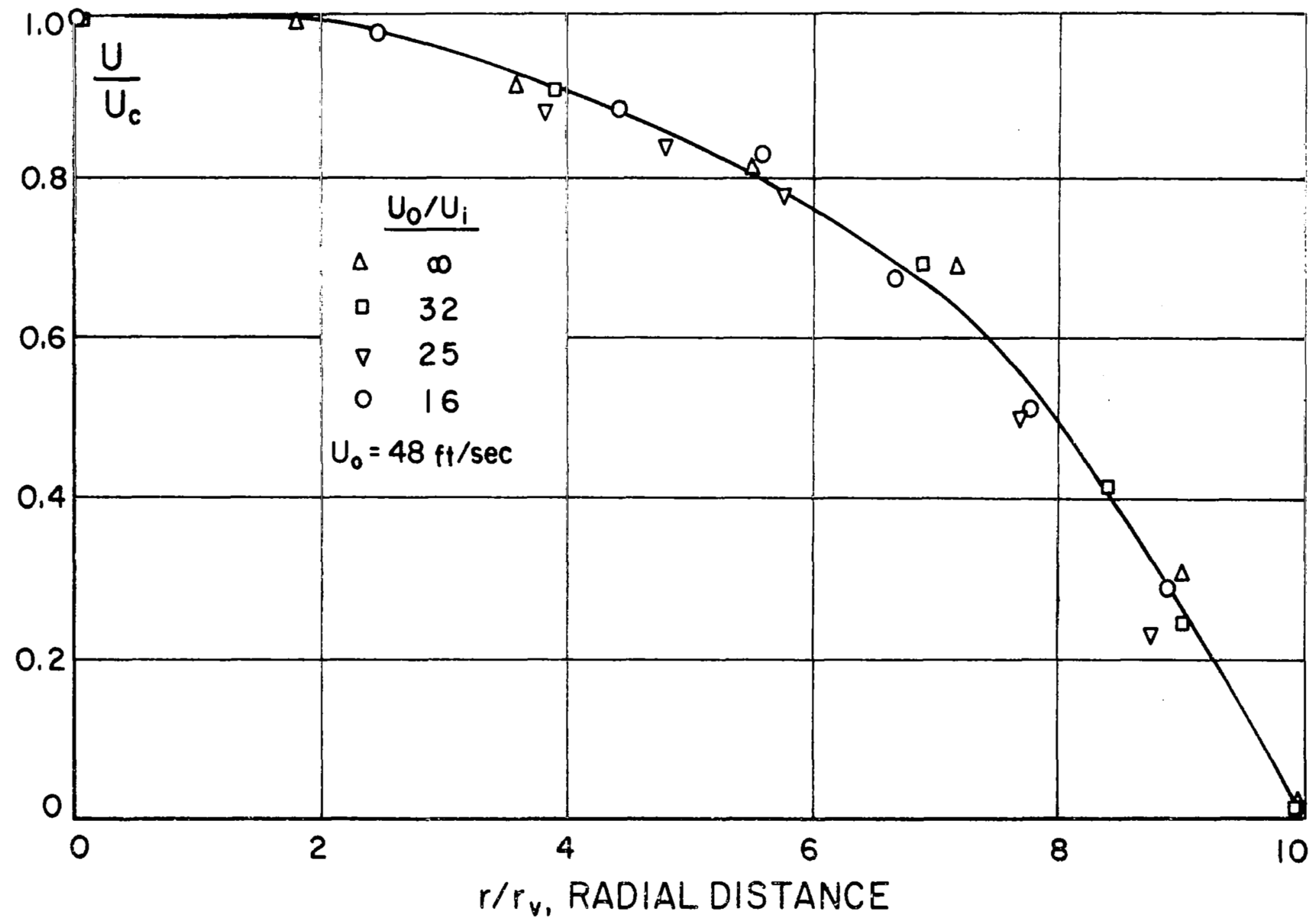

Figure 21. - Radial distribution of nondimensional axial velocity in the vortex. 


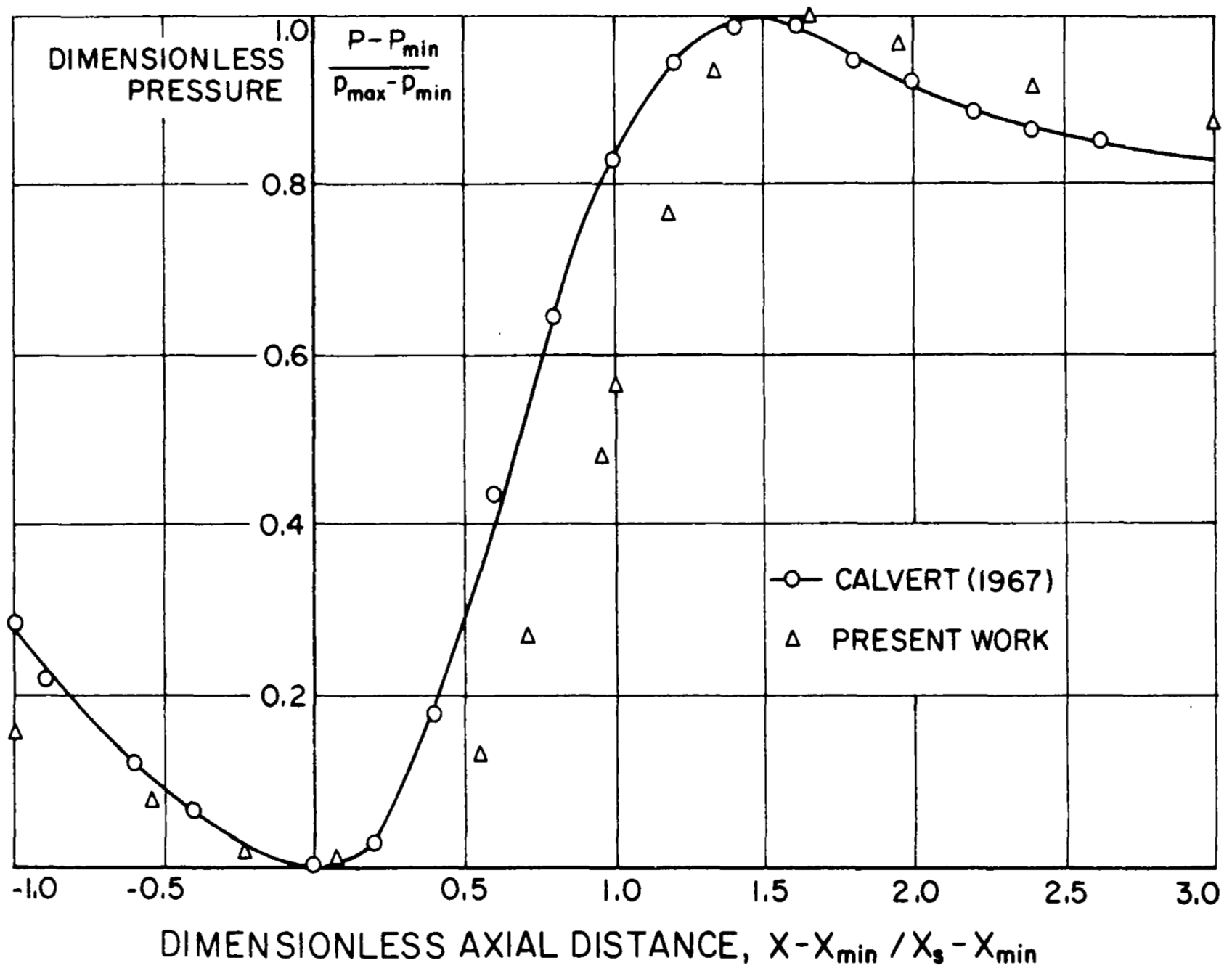

Figure 22. - Correlation of pressure data with results of Calvert (1967). 
zero and the location of the minimum in pressure. The curve shown in figure 22 is the similarity curve for all his pressure data. The pressure curve in the present work for $\mathrm{U}_{\mathrm{o}} / \mathrm{U}_{i}=\infty$ (pure wake) was made non-dimensional using the same pressure and length scales as was used by calvert and the comparison is shown in figure 22. It is shown that although the general shape of the two curves is similar, the correspondence is not good.

In Calvert's work the downstream face of the cone is solid, while for the pure wake in the present work the back flow did penetrate into the pipe. As a result of the difference in Calvert's work the stagnation point is fixed while in the present work the location of the upstream stagnation point is not fixed.

In the coaxial flow case of Johnson (11), a porous plate was attached to the end of the inner stream pipe. This prevented the backflow region from penetrating into the inner stream pipe at high velocity ratios. It therefore is expected that his recirculation region resembled more closely that of Calverts than did that in the present work.

Another comparison is shown of the pressure data with the results reported by Chigier and Beer (16). They measured pressures in the flow region near the nozzle for double concentric jets. No correlation of pressure was attempted by Chigier and Beer. Data that was reported by Chigier and Beer for the case with the annular jet only is compared 
to the pressure measurement in the present work for $\mathrm{U}_{0} / \mathrm{U}_{i}=\infty$. The pressure values were made non-dimensional by plotting $\frac{\mathrm{p}-\mathrm{p}_{\min }}{\mathrm{p}_{\max }-\mathrm{p}_{\min }} \mathrm{v} . \mathrm{s} \cdot \frac{\mathrm{x}-\mathrm{x}_{\min }}{\mathrm{x}_{\max }-\mathrm{x}_{\min }}$ and is shown in figure 23. It is evident that good agreement exists in the region inside the vortex $\left(\frac{x-x_{\min }}{x_{\max }-x_{\min }}\right.$ between zero and one)

The region for which the dimensionless axial distance is greater than 1.0 corresponds to the region downstream of the stagnation point. In this region the agreement between the two experiments is poor since Chigier and Beer used a finite annular jet while in the present case the outer stream can be considered to extend to infinity in the radial direction. It appears that the vortex generated behind a cylindrical pipe in dimensionless variables is independent of the outer stream velocity level. The nondimensional plot of pressure taken for $U_{0}=48 \mathrm{ft} / \mathrm{sec}$ (present work) agreed we11 with measurements taken in a stream of velocity of $45 \mathrm{~m} / \mathrm{sec}$ (Chigier and Beer).

A plot of nondimensional pressure as a function of velocity ratio is presented for the homogeneous case in figure 24 and for the heterogeneous case in figure 25. It is evident that velocity ratio certainly affects the form of the vortex region as is shown through the changing pressure profiles. 


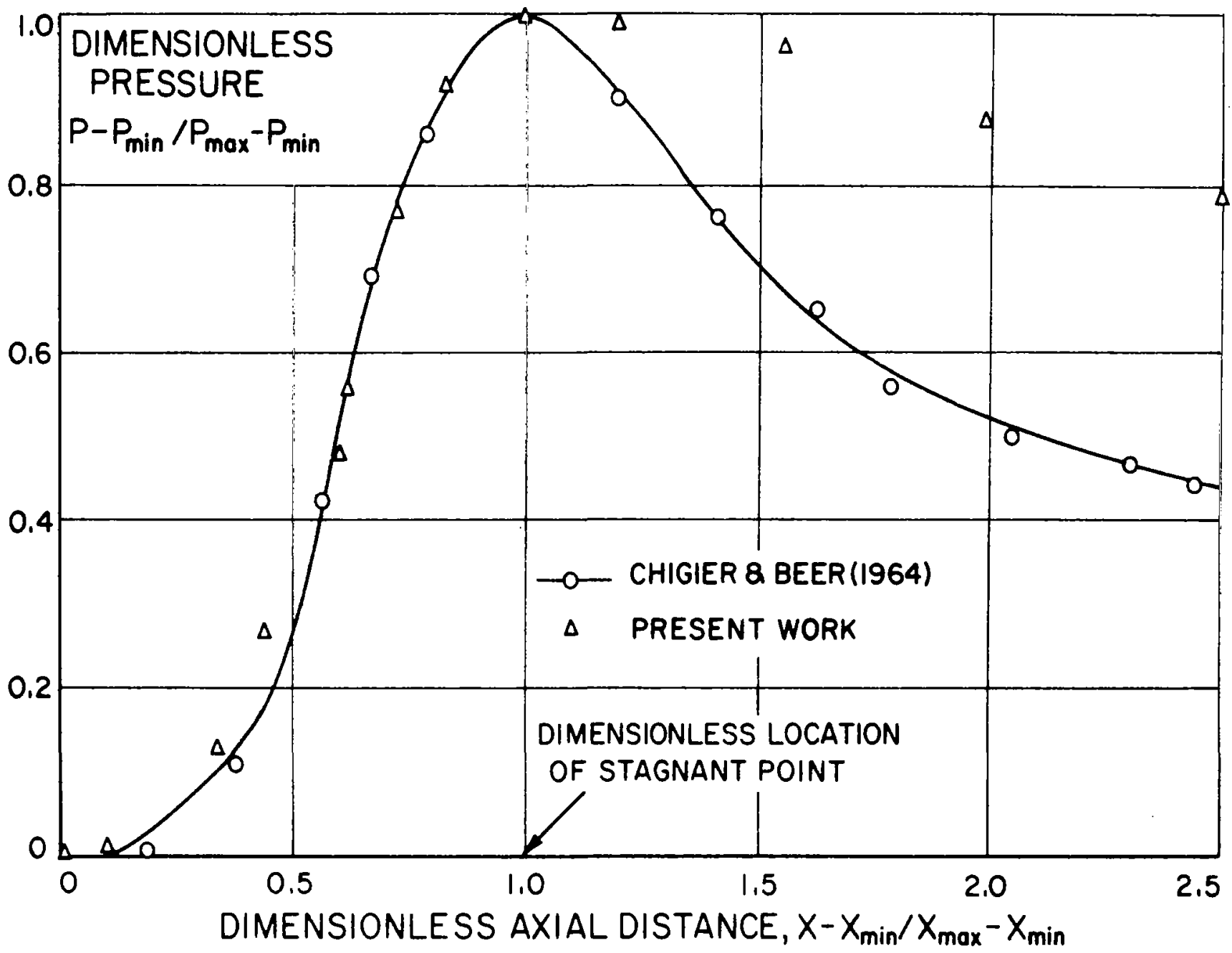

Figure 23. - Correlation of centerline pressure with results given by Chigier and Beer. 
a

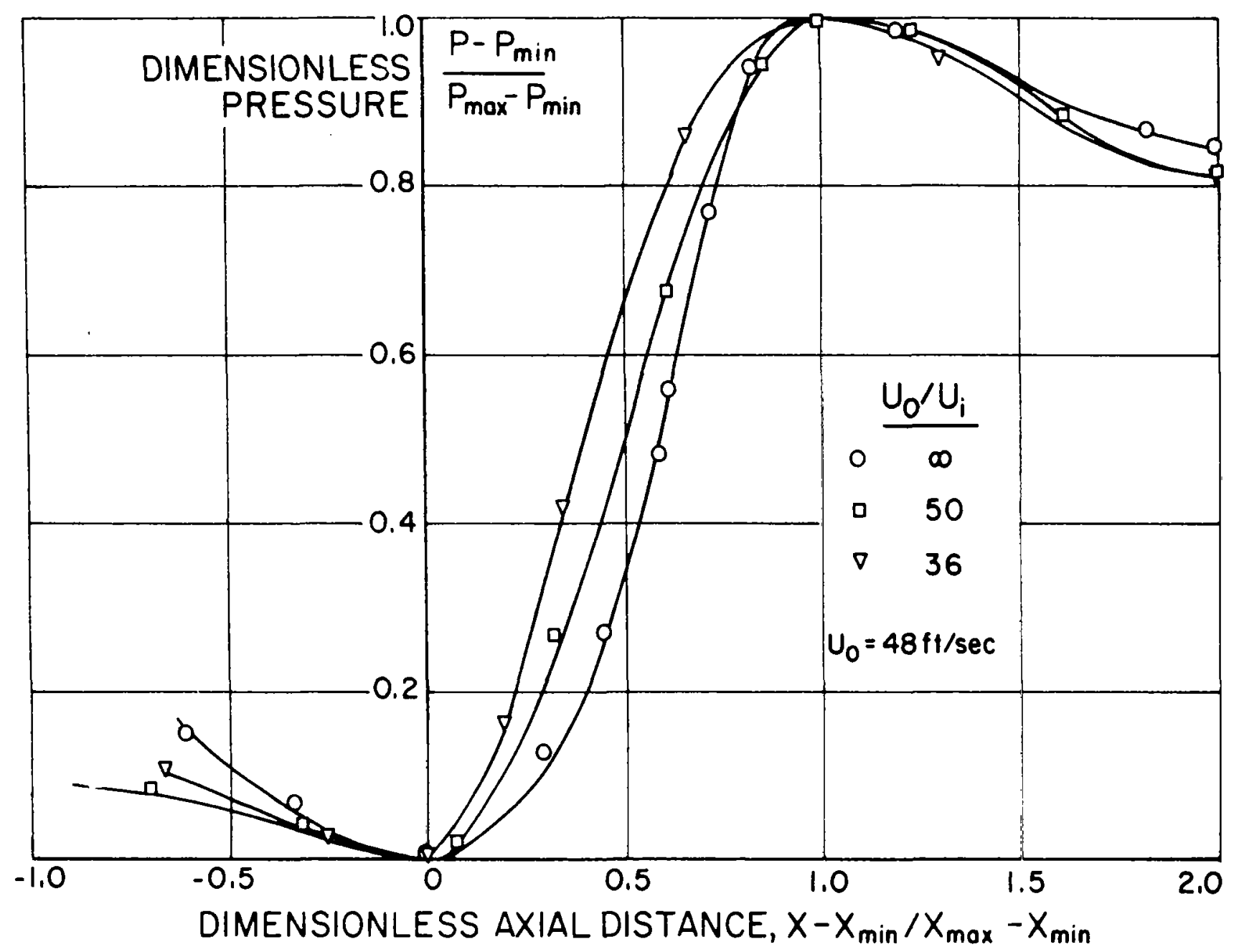

Figure 24. - Homogeneous case. 


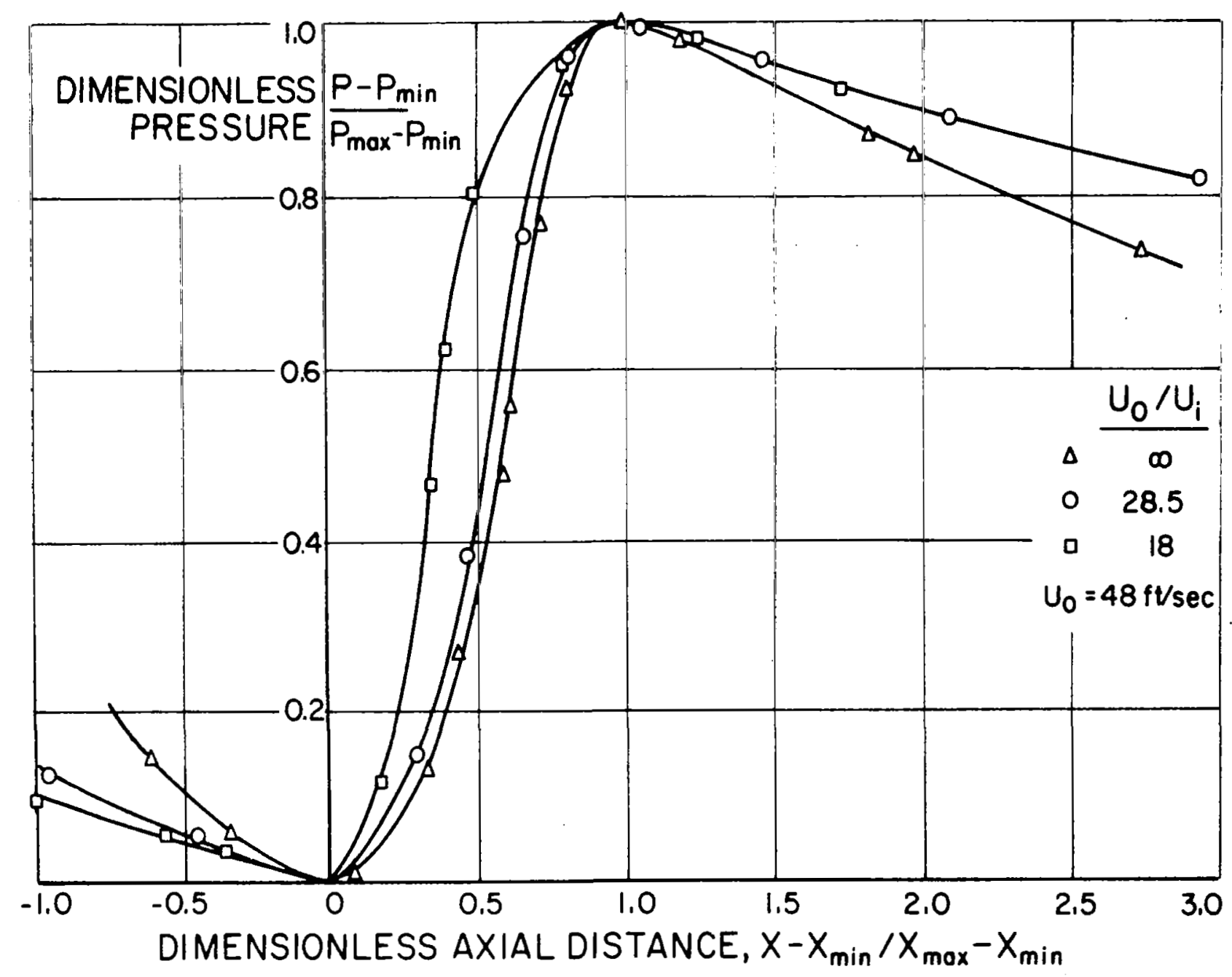

Figure 25. - Heterogeneous case. 


\section{CONCLUSIONS}

1. For $1<\mathrm{U}_{\mathrm{O}} / \mathrm{U}_{i}<4$ the coaxial flow is characterized by a constant momentum deficit at each cross section and an iso-velocity initial potential core.

2. As the velocity ratio $U_{0} / U_{i}$ is increased, the length of the potential core decreases. For the homogeneous case, the potential core disappears at $\mathrm{U}_{\mathrm{O}} / \mathrm{U}_{\mathrm{i}}=3.5$. For the heterogeneous case, the potential core disappears at velocity ratio of approximately $U_{0} / U_{i}=14$. Above this value, the center line velocity decreases first, reaches a minimum, and then increases toward the limiting outer stream velocity. The pressure is not uniform. Initially, the pressure on the center line is lower than free stream pressure. The center line pressure increases further downstream, reaches a maximum, and then drops to the free stream pressure. The location of the maximum in the pressure profile corresponds roughly to the location of the minimum in the velocity profile.

3. A back flow vortex, in the homogeneous case, is formed at velocity ratios, $U_{0} / U_{i}>13$. For the heterogeneous case, the back flow region starts at $\mathrm{U}_{\mathrm{o}} / \mathrm{U}_{i}=26$. The backflow region is at first very unstable, with an intermittent flow component. The backflow vortex becomes more stable when the velocity ratio is increased. For the case of a pure wake $U_{0} / U_{i}=\infty$, the flow patterns are the most stable and the center line pressure and the 
center line turbulence intensity exhibit two peaks corresponding to two stagnation points. The difference between the forward stagnation point pressure and free stream pressure is approximately .12 of the dynamic head of the outer stream. The maximum back flow velocity is $U=-.31 U_{0}$.

4. The pressure field becomes uniform for all velocity ratios downstream of $x / r_{0} \cong 8$. The center line velocity at this location is approximately $U=.5 \mathrm{U}_{\mathrm{O}}$.

5. A non-dimensional form of pressure profile for the pure wake $\left(U_{0} / U_{i}=\infty\right)$ agreed well with a similar profile given by other investigators. This shows that the vortex established in the wake behind a cylinder $\left(U_{0} / U_{i}=\infty\right)$ has self-similar aspects. For velocity ratios between $\mathrm{U}_{\mathrm{O}} / \mathrm{U}_{\mathrm{i}}=(\infty-12)$ for the homogeneous case and $\mathrm{U}_{0} / \mathrm{U}_{i}=$ $(\infty-26)$ for the heterogeneous case, the velocity ratio affects the shape of the wake and nondimensional pressure data did not fall on the same line.

6. Radial distribution of axial velocity at the vortex center cross section for different velocity ratios could be made to fall on the same line with suitable scale parameters; similarly the agreement between nondimensional plot of axial velocity for different velocity ratios was reasonable. 


\section{BIBLIOGRAPHY}

1. Kuethe, A., "Investigation of the Turbulent Mixing Regions Formed by Jets", J.Appl. Mech., 11, 3, A, 87, (1935).

2. Abramovich, G.N., The Theory of Turbulent Jets, MIT Press, Cambridge, (1963).

3. Forstall, W., Jr., and A. H. Shapiro, "Momentum and Mass Transfer in Coaxial Gas Jets", J. Appl. Mech., 17, 4, 399, (1950).

4. Hinze, J.O., and van der Hegge zijnen, Appl. Sci. Research, 1A, 435, 1949.

5. Albertson, M.L., Dai, Y.B., Jensen, R.A., and Rouse, H., "Diffusion of Submerged Jets", Proc. Am. Soc. Civil Engrs., 74, 1571, (1948).

6. Miller, D.R., and Comings, E.W., "Static Pressure Distribution in the Free Turbulent Jet", J.F.M., 3, 1, (1957).

7. Sami, S., Carmody, T., and Rouse, H., "Jet Diffusion in the Region of Flow Establishment", J.F.M., 27, 231, (1967).

8. Trupel, T., "Uber die Einwirkung eines Luftstrahles auf die umgebende Luft", Zeitschrift fur das gesammte Turbinenwesen, $5-6$, (1915).

9. Corrsin, S. and Uberoi, M.S., "Further Experiments on the Flow and Heat Transfer in a Heated Turbulent Air Jet", NACA, 998, (1950). 
10. Zawacki, T.S., and Weinstein, H., "Experimental Investigations of Mixing of Dissimilar Fluids", NASA Contractor Report CR-957, (1968).

11. Johnson, B., "Experimental Study of Multi-Component Coaxial-Flow Jets in Short Chambers", NASA, CR-1190, 1968.

12. Foppl, Sitzungsberichte d.k. bayer, Akad, Wissentschaflicher Gottingen, 1, (1913).

13. Carmody, T., "Establishment of the Wake Behind a Disk", J. Basic Engr., Trans. ASME Series D, 87, 4, 869 (1964).

14. Calvert, J.R., "Experiments on the Low Speed Flow Past Cones", J.F.M.', 27, 273, (1967).

15. Chevray, R., "The Turbulent wake of a Body of Revolution", J. Basic Engr., Trans. of the ASME, 275 (June, 1968).

16. Chigier, N.A., and Beer, J.M., "The Flow Region Near the Nozzle in Double Concentric Jets", J. Basic Engr. Trans. ASME Series D, 37, 4, 823, (1964).

17. Milne-Thompson, J., Theoretical Hydrodynamics, McMillan Press, New York, (1963).

18. Shavit, G., and Lavan, Z., Private communication, (September, 1968).

19. Lavan, Z., and Fejer, A.A., J. Fluid Mech., 23, pt. 1, $173(1965)$.

20. Tmoore, A., "The Effect of Turbulence on Static Pressure Measurements", A.R.C., 22, 010, (1960). 